\title{
Iterative Receiver Design for Polar-Coded SCMA Systems
}

\author{
Luping Xiang, Member, IEEE, Yusha Liu, Chao Xu, Senior Member, IEEE, Robert G. Maunder, Senior Member, \\ IEEE, Lie-Liang Yang, Fellow, IEEE and Lajos Hanzo, Fellow, IEEE
}

\begin{abstract}
An edge-cancellation-aided iterative detection and decoding (EC-IDD) algorithm is proposed for polar-coded sparse code multiple access (SCMA), which jointly performs Gaussianapproximated message passing (GA-MP) detection of SCMA supported by the soft list decoding (SLD) of polar codes. A reduced-edge factor graph is formulated in each consecutive iteration with the aid of the cyclic redundancy check (CRC) and EC. Based on the simplified factor graph, the EC-IDD gradually reduces its complexity in each subsequent iteration, while improving the bit error rate (BER) performance, compared to the state-of-the-art joint detection and decoding (JDD) of polar-coded SCMA. Furthermore, an embedded decision-directed channel estimator (DD-CE) is proposed for our polar-coded SCMA system under realistic imperfect channel state information (CSI). Our simulation results demonstrate that the proposed EC-IDD achieves better BER performance than the state-ofthe-art JDD under both perfect and imperfect CSI, despite achieving a complexity reduction of $92 \%$. Finally, the BER of the proposed joint DD-CE and EC-IDD algorithm under imperfect CSI converges to that of EC-IDD operating under perfect CSI.
\end{abstract}

Index Terms-Sparse code multiple access (SCMA), Gaussianapproximated message passing (GA-MP) detection, iterative detection and decoding (IDD), channel estimation (CE).

\section{INTRODUCTION}

Aiming for improving the spectral efficiency (SE) of nextgeneration systems, diverse non-orthogonal multiple access (NOMA) schemes have been proposed [1-7]. In contrast to orthogonal multiple access (OMA) that has been adopted in $2 \mathrm{G}, 3 \mathrm{G}$ and $4 \mathrm{G}$, by definition, the NOMA technique performs non-orthogonal resource allocation for the sake of increasing the system load by tolerating limited user interference. In [1], 16 different NOMA schemes were surveyed, among which sparse code multiple access (SCMA) [8] is the focus of this work. Recent research aiming for eliminating the bottlenecks of the SCMA scheme in terms of its throughput, latency and complexity can be found in [9-12].

The joint design of a channel decoder and NOMA detector has originally been proposed in [13], which considers the convolutional-coded low density signature orthogonal frequency division multiplexing (LDS-OFDM) system. Following this,

The authors are with School of Electronics and Computer Science, University of Southampton, SO17 1BJ, UK. (E-mail: $1 x 1 \mathrm{~g} 15$, yl6g15, cx1g08, rm, 1ly, lh@soton.ac.uk). (Corresponding author: Yusha Liu).

The financial support of the Engineering and Physical Sciences Research Council project EP/P034284/1 is gratefully acknowledged

L. Hanzo would like to gratefully acknowledge the financial support of the Engineering and Physical Sciences Research Council projects EP/Noo4558/1, EP/PO34284/1, COALESCE, of the Royal Society's Global Challenges Research Fund Grant as well as of the European Research Council's Advanced Fellow Grant QuantCom (Grant No. 789028) several iterative detection and decoding (IDD) algorithms have been proposed for NOMA amalgamated with low-density parity-check (LDPC), turbo or polar codes [14-17]. Compared to LDPC and turbo codes, polar codes are considered as a less mature error correction code, even though it has been standardized for protecting the control channel transmission in 5G New Radio (NR) [18,19]. Following the standardization, joint detection and decoding (JDD) of polar-coded solutions designed for the $5 \mathrm{G}$ control channels have been investigated in [20]. However, the limited throughput and error propagation of the best-performing hard-output successive cancellation list (SCL) decoding have prevented polar coding from finding its way to the $5 \mathrm{G}$ data channel.

Furthermore, belief propagation (BP) was first polar decoder proposed to achieve soft-input soft-output (SISO), suffering from performance degradation compared to the SCL decoder [21], while the state-of-the-art SISO polar decoder, referred to as the soft cancellation (SCAN) decoder [22], achieves a lower decoding complexity compared to the BP decoder. More sophisticated polar decoders have also been proposed [23-28] for achieving low-complexity hard-output decoding, which are capable of attaining the bit error rate (BER) performance of the SCL decoder. By contrast, the family of soft-output polar decoders include SCAN list [29] and $\mathrm{BP}$ list [30] polar decoders, mitigating the performance loss of SCAN or BP decoding. Elaborating further on SISO decoder, we have previously proposed a novel soft list decoder (SLD) [31], which outperforms the hard-output SCL polar decoder, hence facilitating promising applications of the polarcoded SCMA.

Previous work on the JDD of polar-coded systems is summarised in Table I [16, 20,32-38], which allows us the boldly and explicitly contrast our new contributions to the existing state-of-the-art. While these studies designed impressive JDD algorithms, there is a paucity of contributions on the convergence analysis, on the BER performance over fading channels and on complexity reduction techniques. Furthermore, none of these studies integrate channel estimation (CE) seamlessly into the joint receiver design.

Motivated by efficiently exploiting the output information of both the SCMA detector and of the polar decoder, we propose a joint receiver design relying on a low-complexity Gaussianapproximated message passing (GA-MP) detection [39], our previously proposed SISO SLD [31] and the edge cancellation (EC). Additionally, the proposed receiver design is intrinsically amalgamated with decision-directed (DD)-CE [40] under imperfect channel state information (CSI), which achieves a 
TABLE I

OVERVIEW OF EXISTING LITERATURE ON THE POLAR-CODED SYSTEMS.

\begin{tabular}{l|c|c|c|c|c|c|c|c|c|c}
\hline Contributions & Proposed work & [16] & [20] & [32] & [33] & [34] & [35] & [36] & [37] & [38] \\
\hline \hline Joint factor graph design & $\checkmark$ & & & $\checkmark$ & & $\checkmark$ & & & & \\
\hline SISO Receiver & $\checkmark$ & & $\checkmark$ & $\checkmark$ & & $\checkmark$ & $\checkmark$ & $\checkmark$ & & $\checkmark$ \\
\hline User load investigation & $\checkmark$ & & & & $\checkmark$ & & $\checkmark$ & & & \\
\hline BER improvement & $\checkmark$ & & $\checkmark$ & $\checkmark$ & $\checkmark$ & $\checkmark$ & & $\checkmark$ & $\checkmark$ & \\
\hline Fading channels & $\checkmark$ & & $\checkmark$ & & $\checkmark$ & $\checkmark$ & $\checkmark$ & $\checkmark$ & & \\
\hline Complexity reduction & $\checkmark$ & $\checkmark$ & & & & & & $\checkmark$ & $\checkmark$ & $\checkmark$ \\
\hline EXIT chart analysis & $\checkmark$ & & & & $\checkmark$ & $\checkmark$ & & & & \\
\hline Early termination & $\checkmark$ & & & & & & & & & \\
\hline Joint CE & $\checkmark$ & & & & & & & & & \\
\hline
\end{tabular}

similar BER performance to that under perfect CSI. The main contributions of this paper are summarized as follows:

- An edge-cancellation-aided iterative detection and decoding (EC-IDD) algorithm is proposed for polar-coded SCMA, which jointly performs GA-MP detection and SLD. By performing cyclic redundancy check (CRC), a reduced-size factor graph may be formulated by applying the EC technique, which cancels the variable nodes (VNs) in the factor graph corresponding to the decoded bits that successfully pass the CRC in each consecutive iteration. The proposed EC-IDD improves the BER performance, despite gradually reducing the detection complexity in the subsequent iterations. Naturally, early termination is achieved as a benefit of CRC.

- Furthermore, we propose a DD-CE for our polar-coded SCMA system operating under imperfect CSI. The proposed DD-CE selects the most reliable log-likelihood ratios (LLRs) obtained from GA-MP detection, based on which LS CE is performed. In this way, DD-CE does not require an extra loop for performing $\mathrm{CE}$ separately. This is in contrast to the conventional $\mathrm{CE}$, which requires the inner iterative loop for exchanging soft extrinsic information between the detector and channel estimator in each outer iteration. Our simulation results show that the proposed DD-CE achieves significant iterative gain over the conventional Bayesian learning-aided [41] or LS CE [42], and tends to the BER performance recorded under perfect CSI.

- Our convergence rate and BER performance vs. complexity results demonstrate the benefits of our receiver design employing EC-IDD and DD-CE. The convergence behavior of the joint GA-MP detector and SLD is characterised with the aid of EXtrinsic Information Transfer (EXIT) chart for this polar-coded SCMA system for the first time. Furthermore, our polar-coded SCMA receiver is capable of achieving superior BER performance over the state-of-the-art JDD of the polar-coded SCMA system in [34], which employs a message passing aided (MPA) detector and SCAN decoder, despite the significantly lower complexity of our design.

The paper is structured as follows. Section II details the polar-coded SCMA system model, while a novel lowcomplexity iterative receiver is designed for polar-coded SCMA systems in Section III. Following this, the performance of the proposed receiver is characterised in Section IV both under perfect and imperfect CSI and in terms of its convergence, as well as BER vs. complexity. Finally, our conclusions and future research ideas are summarised in Section V.

\section{System Model}

In this section, the transmitter and receiver structure of polar-coded SCMA uplink supporting $K$ users by $N$ orthogonal resources in the frequency domain (FD) is detailed in Sections II-A and II-B, respectively.

\section{A. Transmitter}

The transmitter of the multiuser polar-coded SCMA system uplink is shown in Fig. 1, where each of the $K$ users simultaneously transmits his/her information to a base station (BS) over $N<K$ orthogonal resources in the FD .

To be more specific, within the $q$-th, $q=1, \cdots, Q$, timeslot, the $A$-length information and CRC bits $\boldsymbol{u}_{k}$ transmitted by user $k(1 \leq k \leq K)$ are first interleaved and polar encoded into $E$ encoded bits $\boldsymbol{b}_{k}$, giving a coding rate of $R=A / E$. Here, we assume that each user transmits the same number of information bits in each frame. The $A$-length $\boldsymbol{u}_{k}$ adopts the $A$ most polarised bit channels of the $B=2^{w}(B>A)$ bit channels, which are determined by the 3rd Generation Partnership Project (3GPP) standard of the 5G NR polar codes [18], where $w$ is an integer. The remaining $(B-A)$ bit channels transmit 0 -valued frozen bits. Then the resultant $B$ length vector $\boldsymbol{u}_{k}^{(0)}$ comprising $B$ bits may be polar encoded into a vector $\boldsymbol{u}_{k}^{(w)}$ of $B$ encoded bits [19], according to the modulo-2 matrix multiplication

$$
\boldsymbol{u}_{k}^{(w)}=\boldsymbol{u}_{k}^{(0)} \mathbf{G}_{2}^{\otimes w}
$$

where $\mathbf{G}_{2}^{\otimes w}$ is the generator matrix, and the superscript $\otimes w$ indicates the $w$-th Kronecker power of the matrix $\mathbf{G}_{2}$, which is expressed as

$$
\boldsymbol{G}_{2}=\left[\begin{array}{ll}
1 & 0 \\
1 & 1
\end{array}\right] .
$$

Figure 2 exemplifies this encoding operation, where the core information block is $\boldsymbol{u}_{k}^{(0)}=$ [00000100], with $u_{k}^{(3,0)}, u_{k}^{(5,0)}$, $u_{k}^{(6,0)}$, and $u_{k}^{(7,0)}$ being the information bits, which is input at the left-hand edge of the graph and the $w=3$ successive 


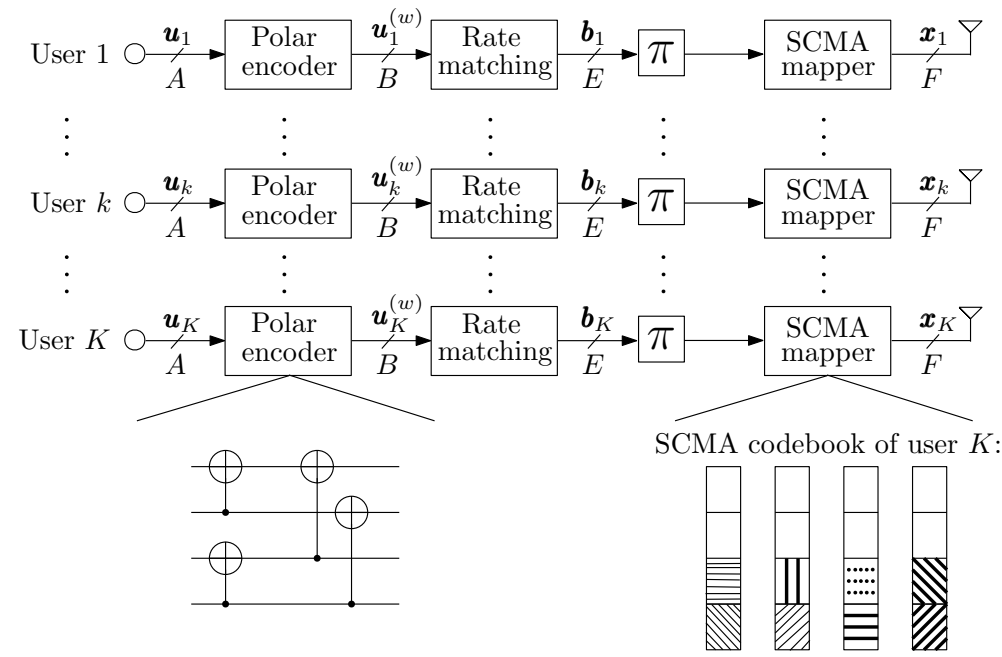

Fig. 1. Transmitter of the polar-coded SCMA system.

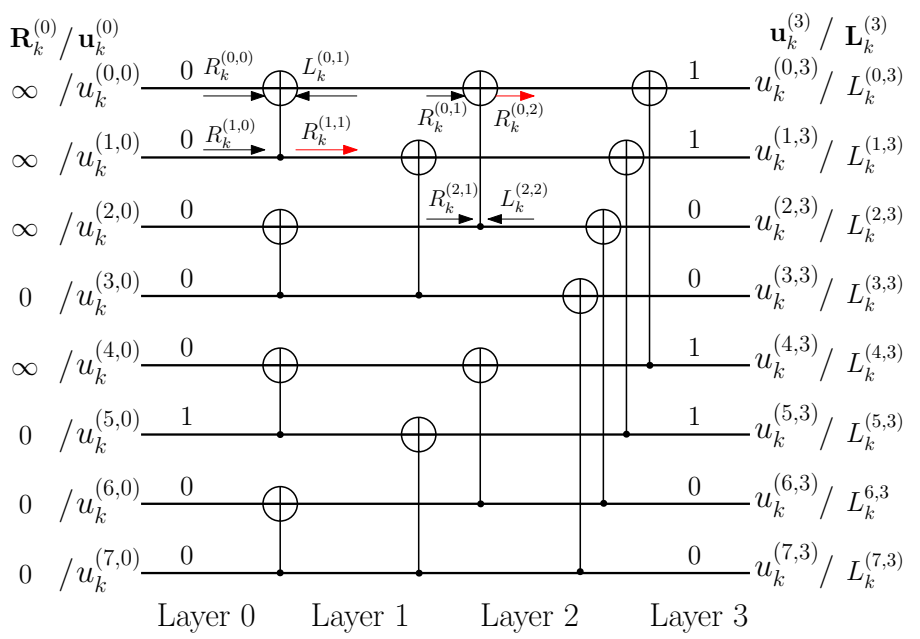

Fig. 2. The code graph example of polar codes, where the core information block $\boldsymbol{u}_{k}^{(0)}=[00000100]$ is converted to the core encoded block $\boldsymbol{u}_{k}^{(3)}=$ [11001100].

layers of XOR operations produce the core encoded block $\boldsymbol{u}_{k}^{(3)}=[11001100]$ on its right-hand edge.

The encoded bits $\mathbf{u}_{k}^{(w)}$ are output from the encoder for subsequent operations, which typically includes rate matching, as in the 3GPP NR uplink polar code [25]. As shown in Fig. 1, after rate matching, a polar-coded block comprising $E$ bits $\boldsymbol{b}_{k}$ is generated.

The polar encoded bits $\boldsymbol{b}_{k}$ are interleaved and then mapped by the SCMA codebook $\mathcal{S}_{k}$ to $F=E / \log _{2} M$ codeword symbols $\boldsymbol{x}_{k}=\left[\boldsymbol{x}_{k, 1}^{T}, \boldsymbol{x}_{k, 2}^{T}, \cdots, \boldsymbol{x}_{k, F}^{T}\right]^{T}$, where $M$ is the modulation order and the cardinality of $\mathcal{S}_{k}$ is $\left|\mathcal{S}_{k}\right|=M$. We also define that the $f$-th SCMA codeword $x_{k n, f}$ of user $k$ transmitted by subcarrier $n$ is selected from the codebook $\mathcal{S}_{k, n}$. Additionally, the positions of the non-zero elements of the $f$ th codeword symbol of all $K$ users $\boldsymbol{X}_{f}=\left[\boldsymbol{x}_{1, f}, \boldsymbol{x}_{2, f} \cdots \boldsymbol{x}_{K, f}\right]$ can be illustrated in a indicator matrix $V[8,43]$, which can be exemplified as

$$
\boldsymbol{V}=\left[\begin{array}{llllll}
1 & 1 & 1 & 0 & 0 & 0 \\
1 & 0 & 0 & 1 & 1 & 0 \\
0 & 1 & 0 & 1 & 0 & 1 \\
0 & 0 & 1 & 0 & 1 & 1
\end{array}\right]
$$

where the $d_{x}=2\left(d_{x} \ll N\right)$ non-zero elements in the $k$ th column, $k=1, \cdots, K$, of $V$ represent the $d_{x}$ subcarriers employed for transmitting the signal of user $k$, while the $d_{c}=$ $3\left(d_{c} \ll K\right)$ non-zero elements in the $n$-th row, $n=1, \cdots, N$, of $V$ represent the $d_{c}$ users who share the same $n$-th subcarrier to transmit their signal. Then the normalized user load $\lambda$ for a $N \times K$ SCMA system may be defined as

$$
\lambda=\frac{K}{N}=\frac{d_{c}}{d_{x}} .
$$

Then the SCMA symbols, $\boldsymbol{X}_{1}, \cdots, \boldsymbol{X}_{F}$, will be transmitted by $N$ subcarriers over wireless channels.

\section{B. Receiver}

The receiver structure of the polar-coded SCMA system is shown in Fig. 3. We assume that each subcarrier experiences Rayleigh fading. Furthermore, we define the channel impulse response (CIR) between the $n$-th subcarrier of the $k$-th user and the BS in the $f$-th symbol duration as $h_{k n}^{(f)}$. Then the $N$-length received signal observations $\boldsymbol{y}_{f}$ in the $f$-th symbol duration of the $q$-th time-slot can be expressed as

$$
\boldsymbol{y}_{f}=\sum_{k=1}^{K} \operatorname{diag}\left(\boldsymbol{h}_{k}^{(f)}\right) \boldsymbol{x}_{k, f}+\boldsymbol{n}_{f},
$$

where $\boldsymbol{h}_{k}^{(f)}=\left[h_{k 1}^{(f)}, h_{k 2}^{(f)}, \cdots, h_{k N}^{(f)}\right], \boldsymbol{y}_{f}, \boldsymbol{x}_{k, f}$ are both $N \times 1$ vectors and $\boldsymbol{n}_{f}$ obeys the zero-mean complex Gaussian distribution with a covariance matrix of $2 \sigma^{2} \boldsymbol{I}_{N}$, expressed as $\mathcal{C N}\left(0,2 \sigma^{2} \boldsymbol{I}_{N}\right)$, where $\boldsymbol{I}_{N}$ is an $N \times N$ diagnoal matrix, $\sigma^{2}=1 /(2 \gamma)$ and $\gamma$ denotes the signal-to-noise ratio (SNR).

The received signal observations of all the $F$ symbols in one frame can be expressed as

$$
\boldsymbol{Y}=\left[\boldsymbol{y}_{1}, \boldsymbol{y}_{2}, \cdots, \boldsymbol{y}_{F}\right] \text {. }
$$




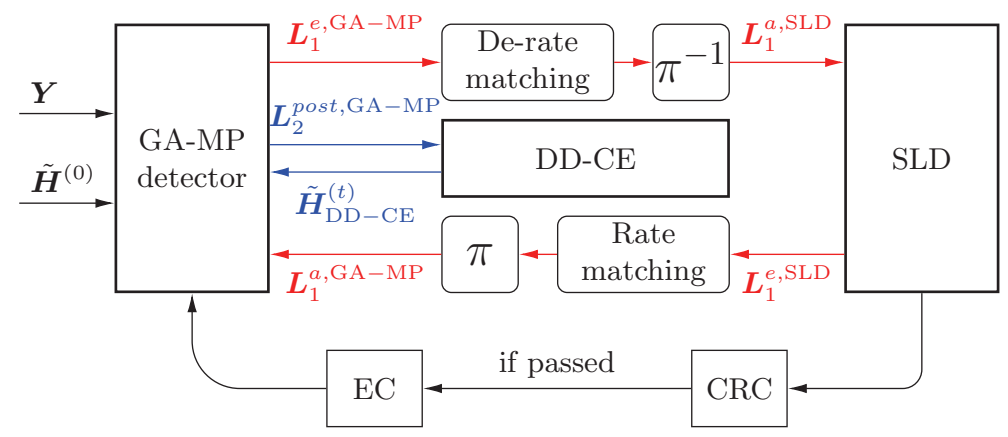

Fig. 3. Proposed receiver design of the polar-coded SCMA system.

As shown in Fig. 3, at the receiver, CE, data detection and decoding are carried out jointly and iteratively, which will be detailed in Section III.

\section{ITERATIVE RECEIVER DESIGN UNDER IMPERFECT CSI}

In this section, we propose a joint receiver design for the polar-coded SCMA system, which iteratively performs GAMP detection, SLD and EC under perfect CSI, as will be detailed in Sections III-A to III-D, and performs embedded DD-CE under imperfect CSI, which will be detailed in Section III-E.

\section{A. GA-MP Detection}

Given the observations $\boldsymbol{y}_{f}$ and either perfect CIRs $\boldsymbol{h}_{k}^{(f)}$ or the initial estimated $\tilde{\boldsymbol{h}}_{k}^{(f)}$ that will be discussed in Section III-E, first, GA-MP detection is applied to the received $\boldsymbol{y}_{f}$, based on the factor graph shown in Fig. 4(a), where the VN $k(1 \leq k \leq K)$ represents the transmit symbol of user $k$, while the check node $(\mathrm{CN}) n(1 \leq n \leq N)$ represents the observations of $n$-th subcarrier at $U$ receive antennas (RAs). For simplicity, we omit the subscript/superscript $f$ when describing GA-MP detection, since the detection operations of all the F SCMA symbols are the same.

Let us first define the connections of the factor graph as a pair of sets, namely the $k$-th $\mathrm{VN}$ to the CNs' connections, and the $n$-th $\mathrm{CN}$ to the VNs' connections, which can be expressed as

$$
\begin{aligned}
& \mathcal{V}_{k}=\left\{n: 1 \leqslant n \leqslant N, e_{k n} \neq 0\right\}, k=1, \cdots, K, \\
& \mathcal{C}_{n}=\left\{k: 1 \leqslant k \leqslant K, e_{k n} \neq 0\right\}, n=1, \cdots, N,
\end{aligned}
$$

where $e_{k n} \neq 0$ represents the connection between the VN $k$ and the $\mathrm{CN} n$.

According to [8], in the $i$-th inner iteration, the message passes via the edges in the factor graph both downwards and upwards and can be updated, respectively, by the following rules:

$\eta_{k, n}^{s_{m},(i)}=\varepsilon_{k, n} \prod_{v \in \mathcal{V}_{k} \backslash n} \delta_{v, k}^{s_{m},(i-1)}, s_{m} \in \mathcal{S}_{k, n}, m=1,2, \ldots, M$

$$
\begin{aligned}
\delta_{n, k}^{s_{m},(i)}= & \sum_{\substack{\boldsymbol{x}_{[n]} \in \mathcal{S}_{\mathcal{C}_{n} \backslash k}, x_{k n}=s_{m}\\
}}\left(\prod_{x_{v n} \in \boldsymbol{x}_{[n]} \backslash x_{k n}} \eta_{k, n}^{x_{v},(i)}\right) \\
& \times p\left(y_{n} \mid \boldsymbol{x}_{[n]}, x_{k n}=s_{m}\right) \\
& s_{m} \in \mathcal{S}_{k, n}, m=1,2, \ldots, M,
\end{aligned}
$$

where $s_{m}$ represent the $m$-th $(1 \leq m \leq M)$ symbol in the symbol set $\mathcal{S}_{k, n}, \eta_{k, n}^{s_{m},(i)}$ represents the information transmitted from the $\mathrm{VN} k$ to the $\mathrm{CN} n$ in the $i$-th inner iteration and where $\varepsilon_{k, n}$ is the normalisation factor ensuring that $\sum_{m=1}^{M} \eta_{k, n}^{s_{m},(i)}=$ 1 , while $\delta_{n, k}^{s_{m},(i)}$ represents information transmitted from the $\mathrm{CN} n$ to the VN $k$ in the $i$-th inner iteration, and $\boldsymbol{x}_{[n]}$ represents the $d_{c}$ symbols sent by the specific $d_{c}$ users who share the $n$-th subcarrier. Additionally, in (7), $\mathcal{V}_{k} \backslash n$ represents the $\left(d_{x}-1\right)$ subcarriers having connections to the user $k$, except for the $n$-th subcarrier, and in (8), $\boldsymbol{x}_{[n]} \backslash x_{k n}$ represents the $\left(d_{c}-1\right)$ symbols transmitted by the $n$-th subcarrier, except for $x_{k n}$. Furthermore, the PDF of $p\left(y_{n} \mid \boldsymbol{x}_{[n]}, x_{k n}=s_{m}\right)$ given $\boldsymbol{x}_{[n]}$ can be expressed as

$$
p\left(y_{n} \mid \boldsymbol{x}_{[n]}\right)=\frac{1}{2 \pi \sigma^{2}} \exp \left(-\frac{\left\|y_{n}-\sum_{k \in \mathcal{C}_{n}} \tilde{h}_{k n} x_{k n}\right\|^{2}}{2 \sigma^{2}}\right),
$$

where $\mathcal{C}_{n}$ given in (6).

However, the computational complexity of (8) increases exponentially with the number of nodes interfering with VN $k$. Therefore, a low-complexity algorithm is required for SCMA detection. The GA-MP formulated in [39] models the information produced by $\mathrm{VN} k$ and by the interferencecontaminated VNs that are connected to $\mathrm{CN} n$ as Gaussian interference. However, for the SCMA system, the number of users interfering with a specific user $k$ is relatively low, hence the approximation of the interference by the Gaussian distribution based on the central limit theorem leads to performance degradation. Therefore, in this paper, we approximate each individual user's signal by a Gaussian distribution and update the mean and variance at each inner iteration following the message passing principles [8]. Specifically, the downward information transmission $\eta_{k, n}^{s_{m},(i)}$ of (7) can be approximated 


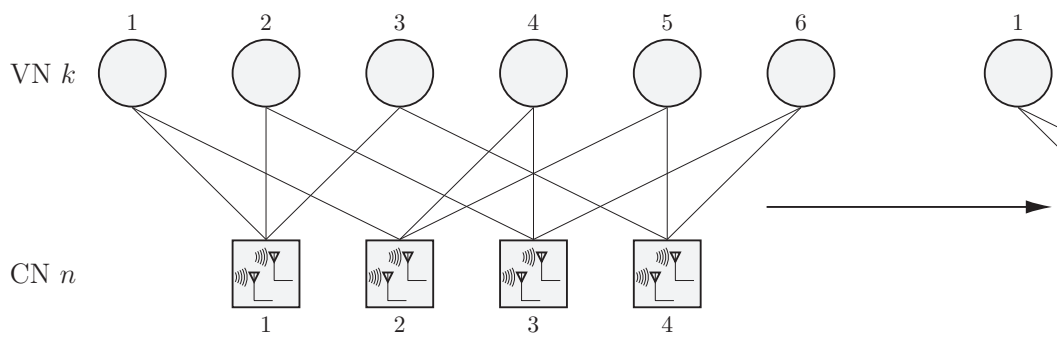

(a)

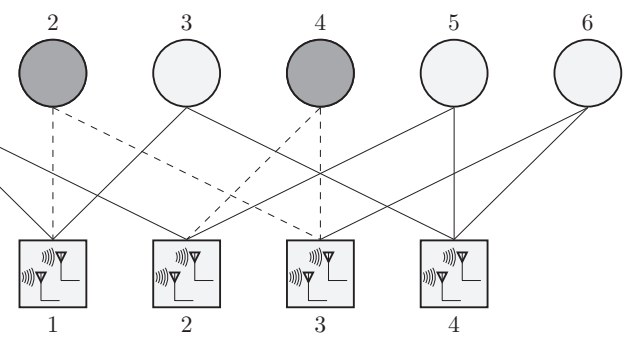

$(b)$

Fig. 4. Factor graph for the (a) $N=4, K=6 \mathrm{SCMA}$ system and (b) edge-cancelled SCMA system during the $t$-th outer iteration.

by a complex Gaussian distribution with a mean of $\bar{x}_{k, n}^{(i)}$ and a variance of $\bar{\tau}_{k, n}^{(i)}$, forming the GA-MP detection.

As shown in Fig. 4, the received signal $\boldsymbol{y}_{f}$ is the input information of the factor graph, and we also have $\eta_{k, n}^{s_{m},(0)}=$ $1 / M$. Then, the mean $\bar{x}_{k, n}^{(0)}$ and variance $\bar{\tau}_{k, n}^{(0)}$ of the variable $s_{m}$ can be initialized as

$$
\begin{aligned}
\bar{x}_{k, n}^{(0)}= & \sum_{s_{m} \in \mathcal{S}_{k, n}} s_{m} \eta_{k, n}^{s_{m},(0)}, k \in \mathcal{C}_{n}, n=1,2, \cdots, N . \\
\bar{\tau}_{k, n}^{(0)}= & \sum_{s_{m} \in \mathcal{S}_{k, n}}\left|s_{m}-\bar{x}_{k, n}^{(0)}\right|^{2} \eta_{k, n}^{s_{m},(0)} \\
& k \in \mathcal{C}_{n}, n=1,2, \cdots, N .
\end{aligned}
$$

As discussed, in the $i$-th $(1 \leq i \leq I)$ inner iteration of GAMP detection, the downward information $\eta_{k, n}^{s_{m},(i)}$ transmitted from the $\mathrm{VN} k$ to the $\mathrm{CN} n$ is approximated by a complex Gaussian function, which can be expressed as

$$
\eta_{k, n}^{s_{m},(i)}=\mathcal{N}_{\mathbb{C}}\left(x_{k n}=s_{m} ; \bar{x}_{k, n}^{(i)}, \bar{\tau}_{k, n}^{(i)}\right) .
$$

Note that the expression of $\bar{x}_{k, n}^{(i)}$ and $\bar{\tau}_{k, n}^{(i)}$ will be discussed later in detail in this section.

Then, the upward transmission $\delta_{n, k}^{s_{m},(i)}$ from the $\mathrm{CN} n$ to the $\mathrm{VN} k$ in the $i$-th GA-MP inner iteration can be calculated as

$$
\begin{aligned}
\delta_{n, k}^{s_{m},(i)}= & \int_{\boldsymbol{x}_{[n]} \in \mathcal{S}_{k}^{d_{c}-1}} p\left(y_{n} \mid \boldsymbol{x}_{[n]}, x_{k n}=s_{m}\right) \\
& \times \prod_{x_{v n} \in \boldsymbol{x}_{[n]} \backslash x_{k n}} \mathcal{N}_{\mathbb{C}}\left(x_{v n}=s_{m} ; \bar{x}_{v, n}^{(i)}, \bar{\tau}_{v, n}^{(i)}\right) .
\end{aligned}
$$

Since $p\left(y_{n} \mid \boldsymbol{x}_{[n]}, x_{k n}=s_{m}\right)$ given by (9) is also Gaussian distributed, (13) can be approximated by a Gaussian function expressed as

$$
\delta_{n, k}^{s_{m},(i)}=\mathcal{N}_{\mathbb{C}}\left(\tilde{h}_{k n} s_{m} ; \bar{\alpha}_{n, k}^{(i)}, \bar{\beta}_{n, k}^{(i)}\right),
$$

where the mean $\bar{\alpha}_{n, k}^{(i)}$ and variance $\bar{\beta}_{n, k}^{(i)}$ are defined as

$$
\bar{\alpha}_{n, k}^{(i)}=y_{n}-\sum_{v \in \mathcal{C}_{n} \backslash k}\left(\tilde{h}_{v n} \bar{x}_{v, n}^{(i)}\right)
$$

and

$$
\bar{\beta}_{n, k}^{(i)}=2 \sigma^{2}+\sum_{v \in \mathcal{C}_{n} \backslash k}\left|\tilde{h}_{v n}\right|^{2} \bar{\tau}_{v, n}^{(i)} .
$$

At the $(i+1)$-st inner iteration, $\eta_{k, n}^{s_{m},(i+1)}$ is updated by the values $\delta_{n, k}^{s_{m},(i)}$ obtained in the $i$-th inner iteration as follows

$$
\begin{aligned}
\eta_{k, n}^{s_{m},(i+1)}= & \varepsilon_{k, n} \prod_{v \in \mathcal{V}_{k} \backslash n} \delta_{v, k}^{s_{m},(i)}, \\
& s_{m} \in \mathcal{S}_{k, n}, m=1,2, \ldots, M,
\end{aligned}
$$

where $\varepsilon_{k, n}$ is the normalisation factor ensuring that $\sum_{m=1}^{M} \eta_{k, n}^{s_{m},(i+1)}=1$.

Now, if we substitute the result of (14) into (17), we have:

$$
=\frac{\eta_{k, n}^{s_{m},(i+1)}}{\sum_{s_{m} \in \mathcal{S}_{k, n}}^{a, \mathrm{GA}-\mathrm{MP}}\left(s_{m, n}^{a, \mathrm{GA}-\mathrm{MP}}\left(s_{m}\right) \mathcal{N}_{\mathbb{C}}\left(x_{k n}=s_{m} ; \bar{\xi}_{k, n}^{(i)}, \bar{\gamma}_{k, n}^{(i)}\right)\right.}
$$

where $\eta_{k, n}^{a, \mathrm{GA}-\mathrm{MP}}\left(s_{m}\right)$ is the a priori symbol likelihood converted from the soft outputs $L_{1}^{e, S L D}$ of the SLD in the previous outer iteration, as shown in Fig. 4, which will be discussed in Section III-C, and where we have

$$
\begin{aligned}
& \bar{\xi}_{k, n}^{(i)}=\bar{\gamma}_{k, n}^{(i)} \sum_{v \in \mathcal{V}_{k} \backslash n} \frac{\tilde{h}_{v k}^{*} \bar{\alpha}_{v, k}^{(i)}}{\bar{\beta}_{v, k}^{(i)}}, \\
& \bar{\gamma}_{k, n}^{(i)}=\left(\sum_{v \in \mathcal{V}_{k} \backslash n} \frac{\left|\tilde{h}_{v k}\right|^{2}}{\bar{\beta}_{v, k}^{(i)}}\right)^{-1} .
\end{aligned}
$$

A popular technique of finding the Gaussian approximation of $\eta_{n, k}^{s_{m},(i)}$ is to minimize the inclusive Kullback-Leibler (KL) divergence of $\operatorname{KL}\left(\eta_{n, k}^{s_{m},(i)} \| \hat{\eta}_{n, k}^{s_{m},(i)}\right)$ [44-46], which gives the expression of $\bar{x}_{k, n}^{(i)}$ and $\bar{\tau}_{k, n}^{(i)}$ as

$$
\begin{aligned}
\bar{x}_{k, n}^{(i)} & =\sum_{s_{m} \in \mathcal{S}_{k, n}} s_{m} \eta_{k, n}^{s_{m},(i)}, \\
\bar{\tau}_{k, n}^{(i)} & =\sum_{s_{m} \in \mathcal{S}_{k, n}}\left|s_{m}-\bar{x}_{k, n}^{(i)}\right|^{2} \eta_{k, n}^{s_{m},(i)} .
\end{aligned}
$$

Substituting $i=0$ into (21) and (22), we obtain expressions of (10) and (11).

After I GA-MP inner iterations, we obtain the SCMA symbol likelihood $\eta_{k}^{\boldsymbol{s}_{m}}$ of user $k$, which may be expressed 
as

$$
\eta_{k}^{\boldsymbol{s}_{m}}=\frac{\eta_{k}^{a, \mathrm{GA}-\mathrm{MP}}\left(\boldsymbol{s}_{m}\right) \mathcal{N}_{\mathbb{C}}\left(\boldsymbol{x}_{k}=\boldsymbol{s}_{m} ; \bar{\xi}_{k}, \bar{\gamma}_{k}\right)}{\sum_{\boldsymbol{s}_{m} \in \mathcal{S}_{k}} \eta_{k}^{a, \mathrm{GA}-\mathrm{MP}}\left(\boldsymbol{s}_{m}\right) \mathcal{N}_{\mathbb{C}}\left(\boldsymbol{x}_{k}=\boldsymbol{s}_{m} ; \bar{\xi}_{k}, \bar{\gamma}_{k}\right)},
$$

where $\eta_{k}^{a, \mathrm{GA}-\mathrm{MP}}\left(\boldsymbol{s}_{m}\right)$ is the a priori symbol probability obtained from the soft outputs $\boldsymbol{L}_{1}^{e, S L D}$ of the SLD in the previous outer iteration as will be discussed in Section III-C, while the mean $\bar{\xi}_{k}$ and variance $\bar{\gamma}_{k}$ of the Gaussian distribution $\mathcal{N}_{\mathbb{C}}\left(\boldsymbol{x}_{k}=\boldsymbol{s}_{m} ; \bar{\xi}_{k}, \bar{\gamma}_{k}\right)$ are expressed as

$$
\begin{aligned}
& \bar{\xi}_{k}=\bar{\gamma}_{k} \sum_{v \in \mathcal{V}_{k}} \frac{\tilde{h}_{v k}^{*} \bar{\alpha}_{v, k}}{\bar{\beta}_{v, k}}, \\
& \bar{\gamma}_{k}=\left(\sum_{v \in \mathcal{V}_{k}} \frac{\left|\tilde{h}_{v k}\right|^{2}}{\bar{\beta}_{v, k}}\right)^{-1} .
\end{aligned}
$$

\section{B. LLR Conversion}

The output probabilities of the GA-MP detector in the $t$ th outer iteration, $t=1, \cdots, T$ are converted to the LLRs and input to the SLD after de-interleaving, as shown in Fig. 3. More specifically, the $z$-th, $z=1,2, \cdots, \log _{2} M$, extrinsic bit LLR $L^{e, \operatorname{GA}-\mathrm{MP}}\left(\hat{b}_{k, f}^{(z)}\right)$ of the $f$-th detected SCMA codeword symbol $\hat{\boldsymbol{x}}_{k, f}$ can be calculated as

$$
\begin{aligned}
L_{1}^{e, \mathrm{GA}-\mathrm{MP}}\left(\hat{b}_{k, f}^{(z)}\right) & =L_{2}^{\text {post }, \mathrm{GA}-\mathrm{MP}}\left(\hat{b}_{k, f}^{(z)}\right)-L_{1}^{a, \mathrm{GA}-\mathrm{MP}}\left(\hat{b}_{k, f}^{(z)}\right) \quad(26 \mathrm{a}) \\
& =\ln \frac{\sum_{\boldsymbol{s}_{m} \in \mathcal{S}_{k}, \hat{b}_{k, f}^{(z)}=0} \eta_{k, f}^{\boldsymbol{s}_{m}}}{\sum_{\boldsymbol{s}_{m} \in \mathcal{S}_{k}, \hat{b}_{k, f}^{(z)}=1} \eta_{k, f}^{\boldsymbol{s}_{m}}}-L_{1}^{a, \mathrm{GA}-\mathrm{MP}}\left(\hat{b}_{k, f}^{(z)}\right),
\end{aligned}
$$

where $\hat{b}_{k, f}^{(z)}=0$ and $\hat{b}_{k, f}^{(z)}=1$ represent that the $z$-th bit of $\boldsymbol{s}_{m} \in \mathcal{S}_{k}$ is 0 and 1 , respectively, and where $L_{2}^{\text {post,GA-MP }}\left(\hat{b}_{k, f}^{(z)}\right)$ represents the a posteriori bit LLRs of $\eta_{k, f}^{\boldsymbol{s}_{m}}$ obtained from (23) and is calculated as the first term of (26b). As shown in Fig. 3, when the extrinsic bit LLRs $L_{1}^{e, \mathrm{GA}-\mathrm{MP}}\left(\hat{\boldsymbol{b}}_{k}\right)=$ $\left[L_{1}^{e, \mathrm{GA}-\mathrm{MP}}\left(\hat{b}_{k, 1}^{(1)}\right), L_{1}^{e, \mathrm{GA}-\mathrm{MP}}\left(\hat{b}_{k, 1}^{(2)}\right), \cdots, L_{1}^{e, \mathrm{GA}-\mathrm{MP}}\left(\hat{b}_{k, F}^{\left(\log _{2} M\right)}\right)\right]^{T}$ of all $F$ symbols transmitted by user $k$ are generated, deinterleaving is performed, which can be expressed as

$$
\boldsymbol{L}_{1}^{a, \mathrm{SLD}}\left(\hat{\boldsymbol{b}}_{k}\right)=\pi^{-1}\left[\boldsymbol{L}_{1}^{e, \mathrm{GA}-\mathrm{MP}}\left(\hat{\boldsymbol{b}}_{k}\right)\right] .
$$

As shown in Fig. 3, de-rate matching is performed on these interleaved extrinsic LLRs $\boldsymbol{L}_{1}^{a, \operatorname{SLD}}\left(\hat{\boldsymbol{b}}_{k}\right)$ and we have the de-rate matched $\boldsymbol{L}_{1}^{a, \mathrm{SLD}}\left(\hat{\boldsymbol{u}}_{k}\right)$, which will be input to the SLD as the $a$ priori information.

\section{Soft List Decoding}

For the consistence of expression, we define $\boldsymbol{L}_{1}^{a, \operatorname{SLD}}\left(\hat{\boldsymbol{u}}_{k}\right)=$ $\boldsymbol{L}_{k}^{(w)}$, which will be the right input to the polar code graph of Fig. 2, and hence the $\rho$-th element of $\boldsymbol{L}_{k}^{(w)}$ is expressed as $L_{k}^{(\rho, w)}$.

To start with, the SLD carries out the Log-SCL decoding of [21], giving $Z$ candidate decoded bit sequences, where $Z$ is defined as the list size. During the Log-SCL decoding, inverse scheduling of the encoding is carried out, commencing from the rightmost layer $\varpi=w$ to the leftmost layer $\varpi=0$,

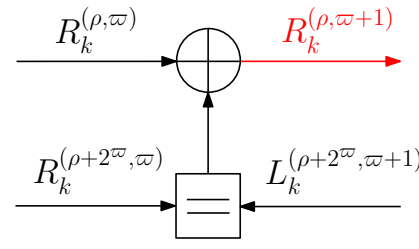

(a)

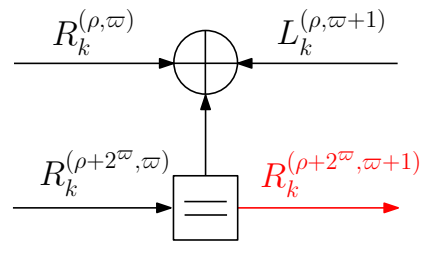

(b)
Fig. 5. LLR propagation of the backwards BP decoding.

as exemplified in Figure 2. For each XOR, three types of computations will be performed, referred to as $f, g$ and partial sum functions ${ }^{1}$, respectively [26]. The decoder also stores both path metrics $(\mathrm{PMs})^{2}$ and LLRs that are input to the $f$ function of each layer $\varpi(\varpi \in[0, w])$ and the intermediate bit sequence $\hat{\boldsymbol{u}}_{k}^{(\varpi)}$ in each layer $\varpi$. After we obtained the $Z$ candidate bit sequences decoded by the Log-SCL decoder, we re-encode the most promising bit sequence $\hat{\boldsymbol{u}}_{k}^{(0)}$, which has the lowest PM, to get hard decisions $\hat{\boldsymbol{u}}_{k}^{(w)}$ at the right-hand side of the polar code graph. The other $(Z-1)$ candidate bit sequences are discarded. At the same time, BP decoding is performed from left to right (backwards) based on the LLRs of the best candidate sequence $\boldsymbol{L}_{k}^{(\varpi)}, \varpi \in[0, w]$, which have been calculated and stored during the previous Log-SCL decoding. The computations of the LLRs during BP decoding are exemplified in Fig. 5.

As shown in Figure 2, since the BP is performed only backwards, we initialise the left-most right LLR of the $\rho$-th bit, $R_{k}^{(\rho, 0)}$, as

$$
R_{k}^{(\rho, 0)}=\left\{\begin{array}{cc}
0, & \text { if } \hat{u}_{k}^{(\rho, 0)} \text { is an information bit; } \\
\infty, & \text { otherwise. }
\end{array}\right.
$$

Following the initialisation and commencing from $\varpi=0$, the right LLRs $R_{k}^{(\rho, \varpi+1)}$ and $R_{k}^{\left(\rho+2^{\varpi}, \varpi+1\right)}$ of the $\rho$-th and $(\rho+$ $\left.2^{\varpi}\right)$-th bit in the $(\varpi+1)$-st layer, respectively, are calculated for all nodes at the $\varpi$-th layer of the code graph as

$$
\begin{gathered}
R_{k}^{(\rho, \varpi+1)}=f\left(R_{k}^{(\rho, \varpi)}, R_{k}^{\left(\rho+2^{\varpi}, \varpi\right)}+L_{k}^{\left(\rho+2^{\varpi}, \varpi+1\right)}\right), \\
R_{k}^{\left(\rho+2^{\varpi}, \varpi+1\right)}=f\left(R_{k}^{(\rho, \varpi)}, L_{k}^{(\rho, \varpi+1)}\right)+R_{k}^{\left(\rho+2^{\varpi}, \varpi\right)} .
\end{gathered}
$$

Note that the left LLRs $L_{k}^{\left(\rho+2^{\varpi}, \varpi+1\right)}$ and $L_{k}^{(\rho, \varpi+1)}$ constitute the inputs of the $f$ functions, which have already been recorded during the right-to-left Log-SCL decoding.

Following backwards BP decoding, the signs of the newly generated right LLRs $\boldsymbol{R}_{k}^{(w)}$ are compared to those of the reencoded bits $\hat{\boldsymbol{u}}_{k}^{(w)}$. If the sign of the LLR $R_{k}^{(\rho, w)}$ is consistent with the $\rho$-th re-encoded bit $\hat{u}_{k}^{(\rho, w)}$, it is entered into the detectors for following the iterative operations. By contrast, the signs of LLRs are flipped but their absolute values remain unchanged, when they are contrary to the polar-encoded bits. Following the LLR flip, the resultant extrinsic LLRs $\boldsymbol{R}_{k}^{e, S L D}$ of the SLD in the $t$-th outer iteration are rate matched to have the

${ }^{1}$ The $f$ function is performed when a pair of LLRs $x$ and $y$ are input to the right-hand side of a XOR, and can be expressed as $f(x, y) \approx$ $\operatorname{sign}(x) \operatorname{sign}(y) \min (|x|,|y|)$. Detailed discussions of $g$ and partial sum functions can be found in [26].

${ }^{2}$ Detailed discussions of PM can be found in [21]. 
$E \times 1$ vector $\overline{\boldsymbol{R}}_{k}^{e, \mathrm{SLD}}$, which is interleaved to give the a priori LLR inputs $\boldsymbol{L}_{1}^{a}, \mathrm{GA-MP}\left(\hat{\boldsymbol{b}}_{k}\right)$ of the GA-MP detector, expressed as

$$
\boldsymbol{L}_{1}^{a, \mathrm{GA}-\mathrm{MP}}\left(\hat{\boldsymbol{b}}_{k}\right)=\pi\left(\overline{\boldsymbol{R}}_{k}^{e, \mathrm{SLD}}\right) .
$$

The interleaved bit LLRs are then re-mapped to symbol likelihoods and will be later entered into the GA-MP detector as the a priori input in the $(t+1)$-st outer iteration, which is expressed as

$$
\begin{aligned}
\eta_{k, f}^{a, \mathrm{GA}-\mathrm{MP}}\left(\boldsymbol{s}_{m}\right)= & \prod_{\substack{\boldsymbol{s}_{m} \in \mathcal{S}_{k}, \hat{b}_{k, f}^{(z)}=0 \\
z \in\left[1, \log _{2} M\right]}} \frac{\exp \left(L_{1}^{a, \mathrm{GA}-\mathrm{MP}}\left(\hat{b}_{k, f}^{(z)}\right)\right)}{1+\exp \left(L_{1}^{a, \mathrm{GA}-\mathrm{MP}}\left(\hat{b}_{k, f}^{(z)}\right)\right)} \\
& \times \prod_{\substack{\boldsymbol{s}_{m} \in \mathcal{S}_{k}, \hat{b}_{k, f}^{(z)}=1 \\
z \in\left[1, \log _{2} M\right]}} \frac{1}{1+\exp \left(L_{1}^{a, \mathrm{GA}-\mathrm{MP}}\left(\hat{b}_{k, f}^{(z)}\right)\right)} .
\end{aligned}
$$

\section{Edge Cancellation}

After the SLD in the $t$-th outer iteration, a CRC is applied to the $Z$ candidate bit sequences of user $k$. In this paper, we employ the $C=11$-bit CRC code standardized by 3GPP Release 16 for the NR physical uplink control channel (PUCCH), with a generator polynomial of $X^{11}+X^{10}+X^{9}+X^{5}+1$. If a candidate successfully passes the CRC, which indicates that the reliability of the current decoded bit sequence is rather high, then the decoded bits will be considered as the final output bits and the corresponding LLRs will no longer be updated during the following outer iterations. Accordingly, both the corresponding $\mathrm{VN} k$ and the edges in the set $\mathcal{V}_{k}$ that have connections to $\mathrm{VN} k$, will be cancelled from the factor graph, as exemplified in Fig. 4. In this way, a CN interacts with a smaller number of VNs in the subsequent iterations, thereby reducing the inter-user interference (IUI). Hence, a reduced-size factor graph will be used for GA-MP detection in the $(t+1)$-st iteration, as shown in Fig. 4(b). Additionally, as and when decoded bit sequences of all $K$ users pass the $\mathrm{CRC}$, all edges of the factor graph are cancelled, hence early termination of the EC-IDD process is achieved.

\section{E. DD-CE Under Imperfect CSI}

In this paper, we adopt the Bayesian estimation, where the CIR taps are random variables having known statistics. Furthermore, we assume that the channel experiences quasistatic Rayleigh fading, which indicates that within the $q$-th time-slot of $F$ symbol durations, the CIR remains constant. We also assume that the $P \ll F$ pilot symbols of each user are known at the receiver, and the $P$ pilots of each user are transmitted independently without IUI. Hence, given the $\left(d_{c} \times P\right)$ received pilots of the $d_{c}$ users that share the $n$-th subcarrier $\check{\boldsymbol{Y}}_{\mathcal{C}_{n}}^{(q)}=\left[\check{\boldsymbol{y}}_{\mathcal{C}_{n}, 1}^{(q)}, \check{\boldsymbol{y}}_{\mathcal{C}_{n}, 2}^{(q)}, \cdots, \check{\boldsymbol{y}}_{\mathcal{C}_{n}, P}^{(q)}\right]$, where $\mathcal{C}_{n}$ is defined in (6) and $\check{\boldsymbol{Y}}^{(q)}=\left[\check{\boldsymbol{Y}}_{\mathcal{C}_{1}}^{(q)}, \check{\boldsymbol{Y}}_{\mathcal{C}_{2}}^{(q)}, \cdots, \check{\boldsymbol{Y}}_{\mathcal{C}_{N}}^{(q)}\right]$, the conventional LS estimator can be employed for estimating the channel gains $\tilde{\boldsymbol{H}}_{\mathcal{C}_{n}}^{(q, 0)}$ between the $n$-th subcarrier and the $d_{c}$ users that share this subcarrier, which aims for minimising $E\left\{\left\|\tilde{\boldsymbol{H}}_{\mathcal{C}_{n}}^{(q, 0)}-\boldsymbol{H}_{\mathcal{C}_{n}}^{(q)}\right\|^{2}\right\}$ by calculating

$$
\tilde{\boldsymbol{H}}_{\mathcal{C}_{n}}^{(q, 0)}=\check{\boldsymbol{Y}}_{\mathcal{C}_{n}}^{(q)} \check{\boldsymbol{X}}_{\mathcal{C}_{n}}^{(q) H}\left(\check{\boldsymbol{X}}_{\mathcal{C}_{n}}^{(q)} \check{\boldsymbol{X}}_{\mathcal{C}_{n}}^{(q) H}\right)^{-1}
$$

where $\check{\boldsymbol{X}}_{\mathcal{C}_{n}}^{(q)}=\left[\check{\boldsymbol{x}}_{\mathcal{C}_{n}, 1}^{(q)}, \cdots \check{\boldsymbol{x}}_{\mathcal{C}_{n}, P}^{(q)}\right]$ represents the $P$ pilot symbols of the users that share the $n$-th subcarrier and $\check{\boldsymbol{X}}^{(q)}=\left[\check{\boldsymbol{X}}_{\mathcal{C}_{1}}^{(q)}, \check{\boldsymbol{X}}_{\mathcal{C}_{2}}^{(q)}, \cdots, \check{\boldsymbol{X}}_{\mathcal{C}_{N}}^{(q)}\right]$. Since we assume that the signals are independent between time-slots, in the following we omit $(q)$ for simplicity.

Instead of implanting an extra CE loop into the system, we propose a DD-CE integrated into the EC-IDD iterations, as shown in Fig. 3, which is inspired by the DD-CE that was originally proposed for multiple-input multiple-output (MIMO) systems in [40,47]. More specifically, after the initial LS CE, we check a pair of conditions for selecting highreliability detected symbols $\hat{\boldsymbol{x}}_{\mathcal{C}_{n}, j}$ for estimating the channel in the next iteration. If the $j$-th symbol of all $d_{c}$ users that share the $n$-th subcarrier satisfy any of our selection conditions, we store the corresponding index $j$ in the set $\mathcal{D}^{(t)}$ for performing the LS CE within each outer iteration. The updated $\tilde{\boldsymbol{H}}_{\mathcal{C}_{n}}^{(t)}$ at the $t$-th iteration will be employed for the EC-IDD in the $(t+1)$-st iteration.

Let us now introduce the a pair of selection conditions for the DD-CE.

Condition 1: The reliability of the $z$-th bit in the $j$-th symbol of all $d_{c}$ users that share the $n$-th subcarrier can be evaluated by the difference of adjacent a posteriori LLRs obtained within a number of inner iterations. If this difference is smaller than a certain threshold, then these LLRs can be considered as reliable ones, and the corresponding bit decisions can be considered as reliable. Hence, they can be employed for the DD-CE of (32) after their conversion to estimated symbols, and they can continue to act as training symbols. This condition can be expressed as

$$
\begin{aligned}
& \frac{\left|L_{2}^{\text {post }, \mathrm{GA}-\mathrm{MP}}\left(\hat{b}_{k, j}^{(z, 1)}\right)-L_{2}^{\text {post }, \mathrm{GA}-\mathrm{MP}}\left(\hat{b}_{k, j}^{(z, 2)}\right)\right|}{|\mu|} \\
& +\cdots+\frac{\left|L_{2}^{\text {post }, \mathrm{GA}-\mathrm{MP}}\left(\hat{b}_{k, j}^{(z, I-1)}\right)-L_{2}^{\text {post }, \mathrm{GA}-\mathrm{MP}}\left(\hat{b}_{k, j}^{(z, I)}\right)\right|}{|\mu|} \leq \phi \\
& \forall k \in \mathcal{C}_{n}, \forall z=1,2, \cdots, \log _{2} M
\end{aligned}
$$

where $\mu$ is the mean of the LLRs $L_{2}^{\text {post,GA-MP }}\left(\hat{b}_{k, j}^{(z, i)}\right), i \in[1, I]$ obtained during the $I$ GA-MP iterations and $\phi$ is the predefined selection threshold.

Condition 2: If the absolute values of the a posteriori LLRs $\left|L_{2}^{\text {post, GA-MP }}\left(\hat{b}_{k, j}^{(z, i)}\right)\right|, i \in[1, I]$ of the $z$-th bit of all $d_{c}$ users sharing the $n$-th subcarrier within a number of consecutive inner iterations are monotonically increasing and have the same sign, this implies that these LLR decisions experience iteration gains. Hence, this bit can be considered as a reliably detected one in the $t$-th iteration and hence can be employed for DD-CE after conversion to the corresponding estimated symbol. This condition is the complement of Condition 1, since a reliable bit can be missed if the consecutive iteration gains lead to significant increase of LLRs. This condition can 
be expressed as

$$
\begin{aligned}
& \left|L_{2}^{\text {post }, \mathrm{GA}-\mathrm{MP}}\left(\hat{b}_{k, j}^{(z, 1)}\right)\right|<\left|L_{2}^{\text {post }, \mathrm{GA}-\mathrm{MP}}\left(\hat{b}_{k, j}^{(z, 2)}\right)\right|<\cdots \\
& <\left|L_{2}^{\text {post }, \mathrm{GA}-\mathrm{MP}}\left(\hat{b}_{k, j}^{(z, I)}\right)\right| \text { andsign }\left\{L_{2}^{\text {post }, \mathrm{GA}-\mathrm{MP}}\left(\hat{b}_{k, j}^{(z, 1)}\right)\right\} \\
= & \operatorname{sign}\left\{L_{2}^{\text {post }, \mathrm{GA}-\mathrm{MP}}\left(\hat{b}_{k, j}^{(z, 2)}\right)\right\}=\cdots=\operatorname{sign}\left\{L_{2}^{\text {post }, \mathrm{GA}-\mathrm{MP}}\left(\hat{b}_{k, j}^{(z, I)}\right)\right\} .
\end{aligned}
$$

If the LLRs of all $\log _{2} M$ bits of all $d_{c}$ users sharing the $n$-th subcarrier satisfy either of these two conditions, then the corresponding index $j$ of $\hat{\boldsymbol{X}}_{j}$ is selected for storing in a set $\mathcal{D}^{(t)}$. Then these LLRs are converted to the symbol probabilities $\eta_{k, j}^{\boldsymbol{s}_{m}}, \quad k \in \mathcal{C}_{n}$ using (31), based on which hard decisions will be carried out using (21). The resultant highreliability symbols can be expressed as

$$
\hat{\boldsymbol{X}}_{\mathcal{C}_{n}, \mathcal{D}^{(t)}}=\left[\hat{\boldsymbol{X}}_{\mathcal{C}_{n}, j_{1}}, \hat{\boldsymbol{X}}_{\mathcal{C}_{n}, j_{2}}, \cdots, \hat{\boldsymbol{X}}_{\mathcal{C}_{n}, j_{D}}\right], j_{i} \in \mathcal{D}^{(t)} .
$$

The selected symbols $\hat{\boldsymbol{X}}_{\mathcal{C}_{n}, \mathcal{D}^{(t)}}$ will then be employed for LS CE relying on (32) to estimate the channels between the $n$-th subcarrier and $d_{c}$ users sharing this subcarrier, together with the pilot symbols $\check{X}_{\mathcal{C}_{n}}$, which can be expressed as

$$
\tilde{\boldsymbol{h}}_{n, \mathrm{DD}-\mathrm{CE}}^{(t)}=\boldsymbol{Y}_{n, \mathcal{D}^{\prime(t)}} \boldsymbol{X}_{\mathcal{C}_{n}, \mathcal{D}^{\prime(t)}}^{H}\left(\boldsymbol{X}_{\mathcal{C}_{n}, \mathcal{D}^{\prime(t)}} \boldsymbol{X}_{\mathcal{C}_{n}, \mathcal{D}^{\prime}(t)}^{H}\right)^{-1}
$$

where $\boldsymbol{X}_{\mathcal{C}_{n}, \mathcal{D}^{\prime(t)}}=\left[\check{\boldsymbol{X}}_{\mathcal{C}_{n}}, \boldsymbol{X}_{\mathcal{C}_{n}, \mathcal{D}^{(t)}}\right], \quad \boldsymbol{Y}_{n, \mathcal{D}^{\prime}(t)}=$ $\left[\check{y}_{\mathcal{C}_{n}, 1}^{\prime}, \cdots, \check{y}_{\mathcal{C}_{n}, P}^{\prime}, \boldsymbol{Y}_{n, \mathcal{D}^{(t)}}\right]$ with $\check{y}_{\mathcal{C}_{n}, p}^{\prime}(1 \leq p \leq P)$ representing the sum of $d_{c}$ elements in $\check{\boldsymbol{y}}_{\mathcal{C}_{n}, p}^{\prime}$ and $\boldsymbol{Y}_{n, \mathcal{D}^{(t)}}$ represents the sub-matrix of $\boldsymbol{Y}_{n}=\left[\boldsymbol{y}_{n, 1}, \cdots \boldsymbol{y}_{n, F}\right]$ with column indices in $\mathcal{D}^{(t)}$. Hence, we have

$$
\tilde{\boldsymbol{H}}_{\mathrm{DD}-\mathrm{CE}}^{(t)}=\left[\tilde{\boldsymbol{h}}_{1, \mathrm{DD}-\mathrm{CE}}^{(t)}, \tilde{\boldsymbol{h}}_{2, \mathrm{DD}-\mathrm{CE}}^{(t)}, \cdots, \tilde{\boldsymbol{h}}_{N, \mathrm{DD}-\mathrm{CE}}^{(t)}\right] .
$$

Finally, the proposed joint DD-CE and EC-IDD algorithm is summarized in Algorithm 1.

\section{PERFormanCE RESUlts}

In this section, we characterize the performance of our proposed receiver design for transmission over quasi-static Rayleigh fading channels, where the channel experiences frequency-flat fading and the channel CIR remains constant within a single frame. More specifically, the convergence behavior, BER performance as well as computational complexity will be quantified in Sections IV-A to IV-C, respectively.

\section{A. Convergence Analysis}

Firstly, we analyse the convergence behavior of both the GA-MP detector and the SLD with the aid of EXIT charts, which constitute a powerful tool of visualizing the convergence behaviour of the IDD process. More specifically, the EXIT chart visualizes the average mutual information (MI) exchange between the concatenated detector and decoder. This is achieved by modeling the associated a priori information of the SCMA detector and polar decoder, respectively, using an independent Gaussian distribution. More details of the EXIT chart construction can be found in [48].

Fig. 6 shows the EXIT chart of the GA-MP detector for the polar-coded SCMA system supporting different number of users after 4 inner iterations and the SLD with $Z=4$ when

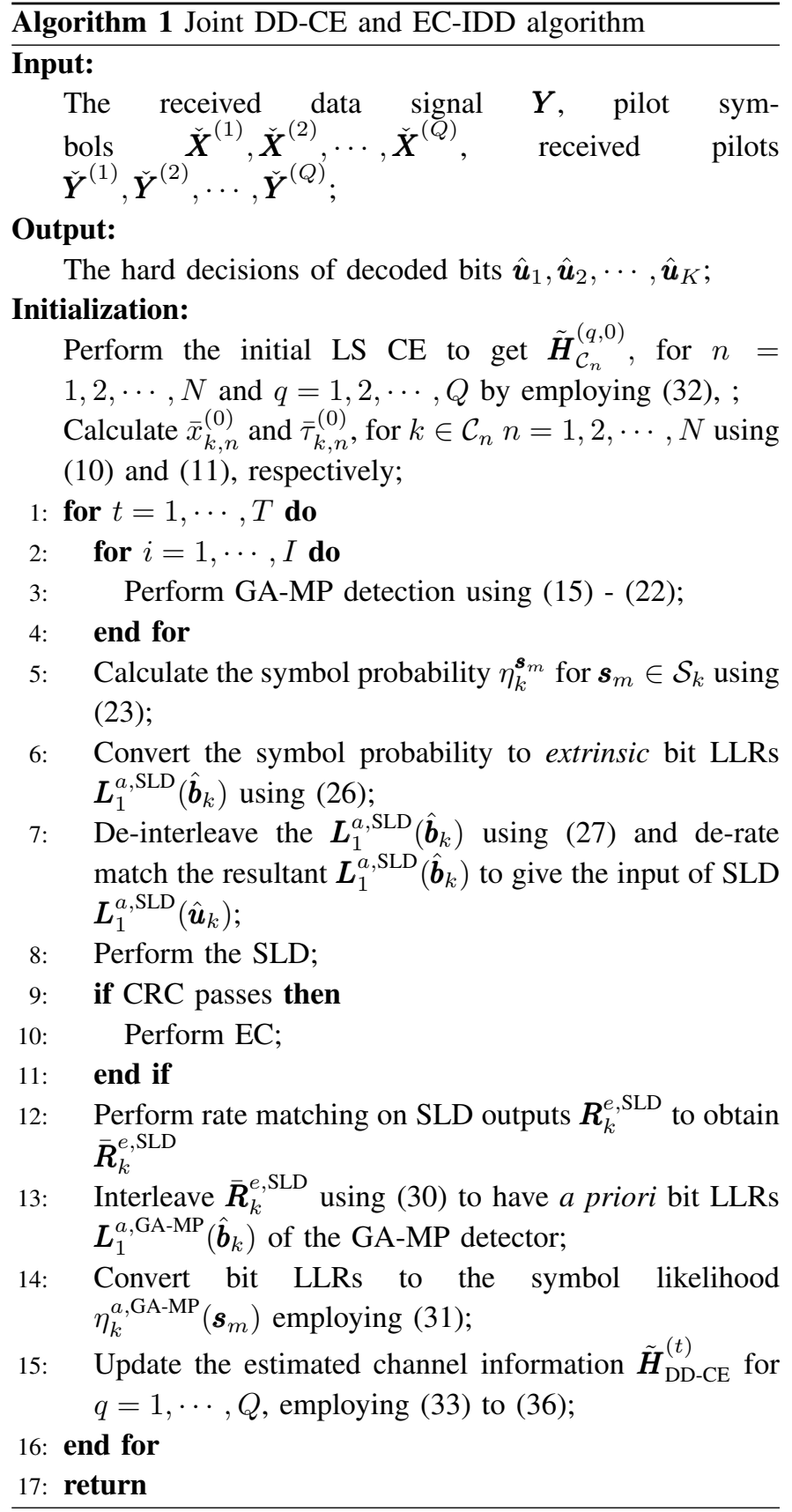

communicating over uncorrelated Rayleigh fading channels. We can see that a higher user load gives a lower initial MI due to the existence of IUI, but different user loads converge to the same point, when the a priori information input to the GA-MP detector reaches 1 . This is because the IUI can be perfectly eliminated, when perfect a priori information is available. This also explains the horizontal EXIT curve for $K=1$, where no IUI exists.

The EXIT chart of the EC-aided GA-MP detection of $K=$ 32 is also presented in Fig. 6, where the same initial point can be observed as for GA-MP detection of $K=32$, but after the $\mathrm{EC}$, a faster convergence to the final MI can be seen, since the IUI is gradually mitigated after the cancellation of highly reliable users. 


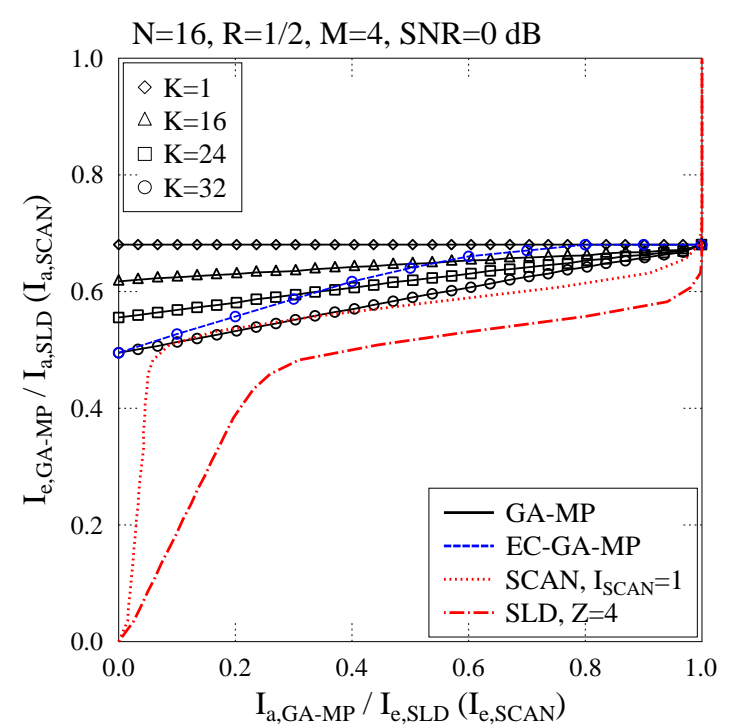

Fig. 6. EXIT chart of the GA-MP detector, SCAN, SLD and EC-aided GA-MP detector.

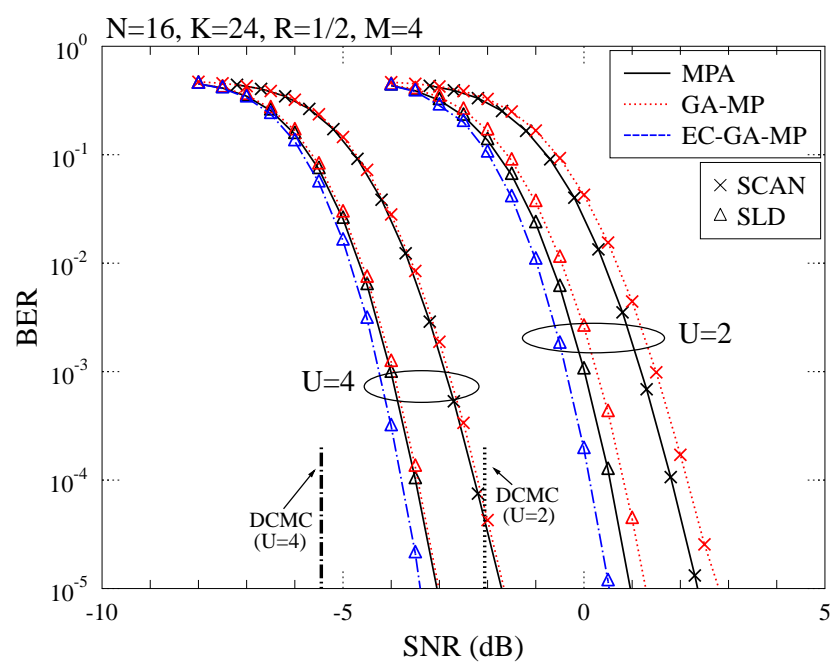

Fig. 7. BER performance of the proposed EC-IDD and the state-of-theart JDD (MPA-SCAN) [34] for polar-coded SCMA system employing $U=$ 2 or 4 RAs and supporting $K=24$ users with $N=16$ subcarriers for communication over quasi-static Rayleigh fading channels.

\section{B. BER Performance}

In this section, we compare the BER performance of our proposed receiver design to that of the state-of-the-art JDD for our polar-coded SCMA system under both perfect and imperfect CSI. In the simulations, we consider a $N=16$, half-rate polar-coded SCMA system, where $F=Q=128$, $M=4$ and the design of the SCMA codebook follows [43]. Also, the number of inner iterations for GA-MP detection is $I=4$ and the number of outer iterations for the EC-IDD and DD-CE is $T=8$. Furthermore, to combat frame-wise fading, the SCMA symbols transmitted by each subcarrier over the $Q$ time-slots are input to a frame interleaver before transmitting over the quasi-static Rayleigh fading channels, which are de-

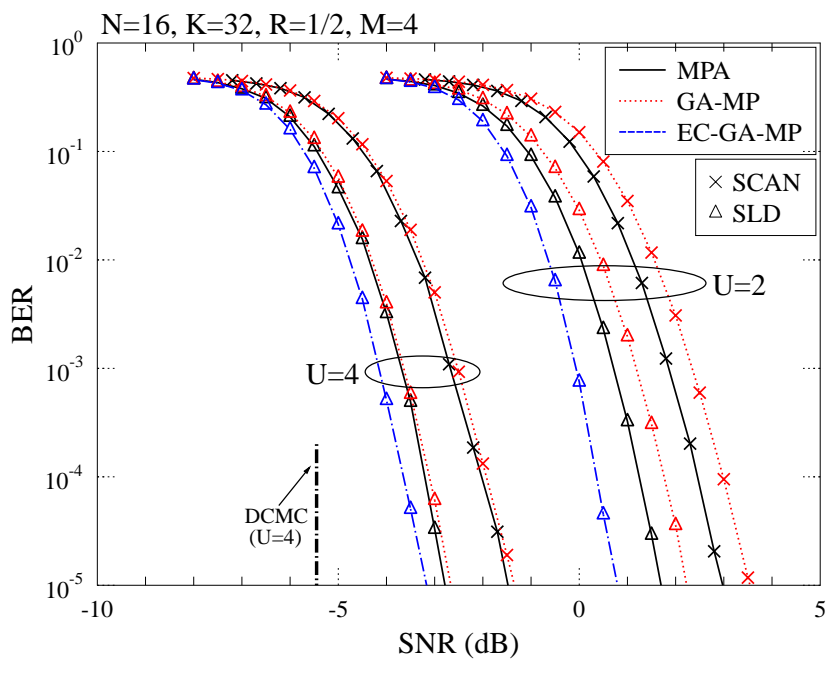

Fig. 8. BER performance of the proposed EC-IDD and the state-of-theart JDD (MPA-SCAN) [34] for polar-coded SCMA system employing $U=$ 2 or 4 RAs and supporting $K=32$ users with $N=16$ subcarriers for communication over quasi-static Rayleigh fading channels.

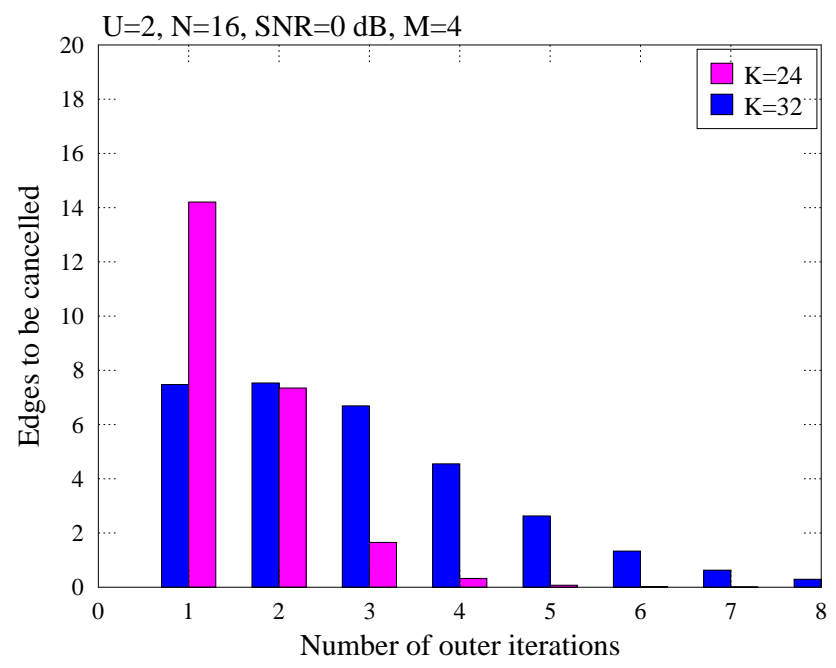

Fig. 9. The number of edges that are cancelled during the EC-IDD of the polar-coded SCMA system supporting $K=24$ and 32 users at $\mathrm{SNR}=0 \mathrm{~dB}$, where $N=16$ subcarriers and $U=2$ RAs are employed.

interleaved at the receiver.

Firstly, Figs. 7 and 8 show the BER comparison of the proposed EC-IDD to the state-of-the-art JDD scheme (MPASCAN) [34] for the normalized user loads of $\lambda=150 \%$ and $200 \%$, respectively, under perfect CSI, where $U=2$ or 4 RAs are employed. We also substitute the MPA detection by the GA-MP as a low-complexity version of the JDD proposed in [34]. Since the CRC appending results in marginal performance gain $[29,34]$, we do not consider the EC-aided detection for SCAN polar decoder in Figs. 7 and 8. Note that the discrete-input continuous-output memoryless channel (DCMC) capacity [49] of the half-rate coded SCMA system is also shown in Fig. 7 as a benchmark of the system. As shown in Figs. 7 and 8, the direct application of GA-MP 


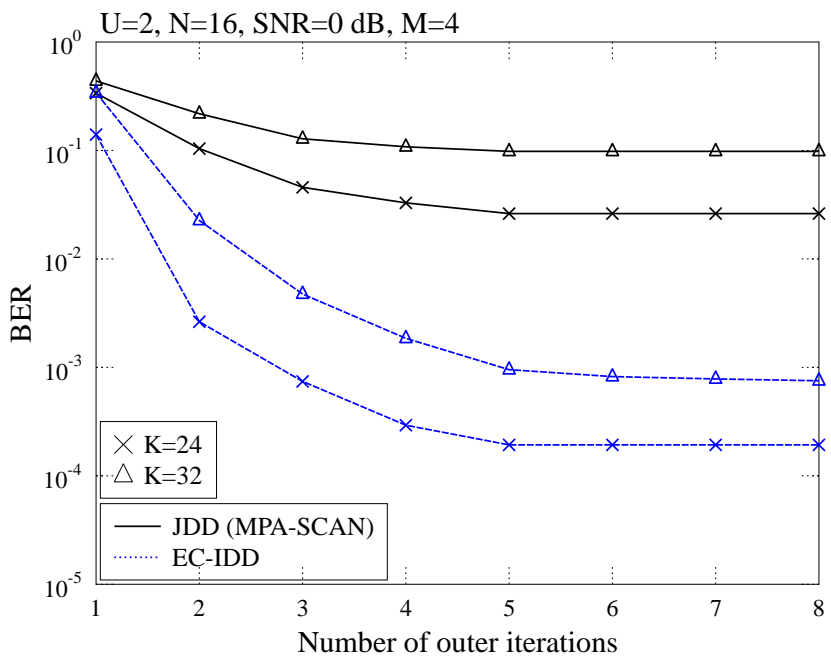

Fig. 10. The BER at $\mathrm{SNR}=0 \mathrm{~dB}$ during each iteration of the EC-IDD and the JDD (MPA-SCAN) of [34] for the polar-coded SCMA system supporting $K=24$ and 32 users at $\mathrm{SNR}=0 \mathrm{~dB}$, where $N=16$ subcarriers and $U=2$ RAs are employed.

detection slightly degrades the BER performance compared to the state-of-the-art MPA detection, when the same polar decoder is employed. By contrast, as shown in Fig. 8, for the polar-coded SCMA system employing GA-MP detection, $1.2 \mathrm{~dB}$ gain can be observed at a BER of $10^{-5}$ when SLD is employed, compared to that employing the SCAN polar decoder. When EC is adopted, a further $0.8 \mathrm{~dB}$ can be attained. Observe also from Fig. 7 that overall, a $1.8 \mathrm{~dB}$ gain can be achieved at a BER of $10^{-5}$ when employing the proposed EC-IDD for $\lambda=150 \%$ compared with the JDD of [34].

In order to better characterize the EC process during the iterative detection, Fig. 9 shows the number of edges that are cancelled in each outer iteration, where $K=16,24$ and 32 users are supported by $N=16$ subcarriers. Explicitly, a smaller $\lambda$ leads to more reliable detection and decoding results, hence a higher number of edges can be cancelled first, whereas a higher normalized user load requires more iterations to attain ultra-reliable BER results that can pass the CRC. This normalized user load vs. reliability trade-off can also be observed in Fig. 10, which shows the BER at $\mathrm{SNR}=0 \mathrm{~dB}$ during each iteration of the EC-IDD and the JDD of [34] for the polar-coded SCMA system with the same parameters as in Fig. 9.

Finally, we investigate the BER performance of the polarcoded SCMA under imperfect CSI. Before quantifying the system performance of the DD-CE, the influence of $\phi$ in the DD-CE on the BER performance is characterized in Fig. 11, where a polar-coded SCMA system $N=16$ and $K=24$ communicating over quasi-static Rayleigh fading channels under imperfect CSI at SNR=0 dB is considered. We can see that $\phi=0.8$ achieves the best BER performance and hence it is selected for the following simulation results considering DD-CE.

The power of embedding DD-CE into our EC-IDD under the imperfect CSI scenario is demonstrated in Figs. 12 and 13,

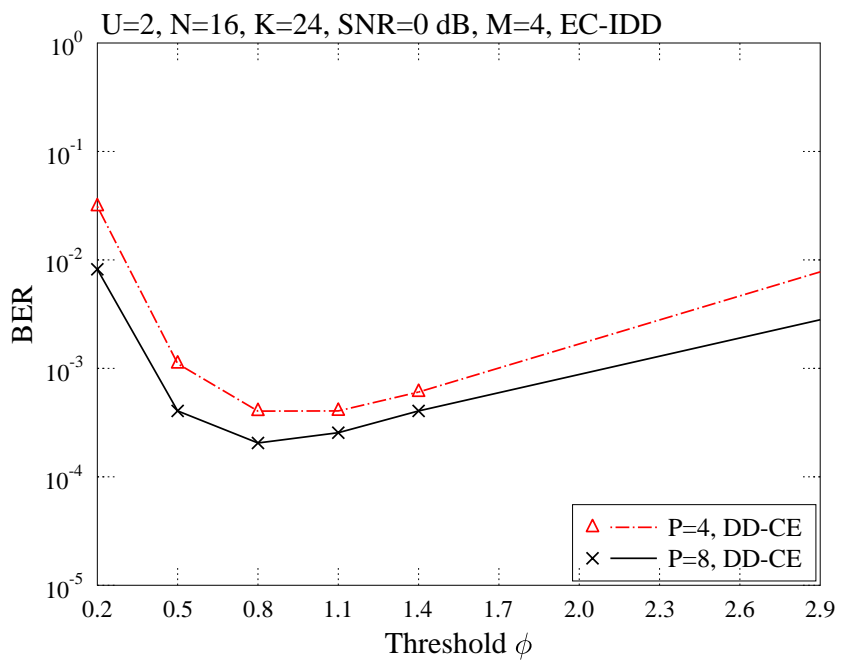

Fig. 11. BER performance the proposed DD-CE employing different $\phi$ s for a $N=16, K=24$ polar-coded SCMA system for communication over quasi-static Rayleigh fading channels under imperfect CSI at $\mathrm{SNR}=0 \mathrm{~dB}$.

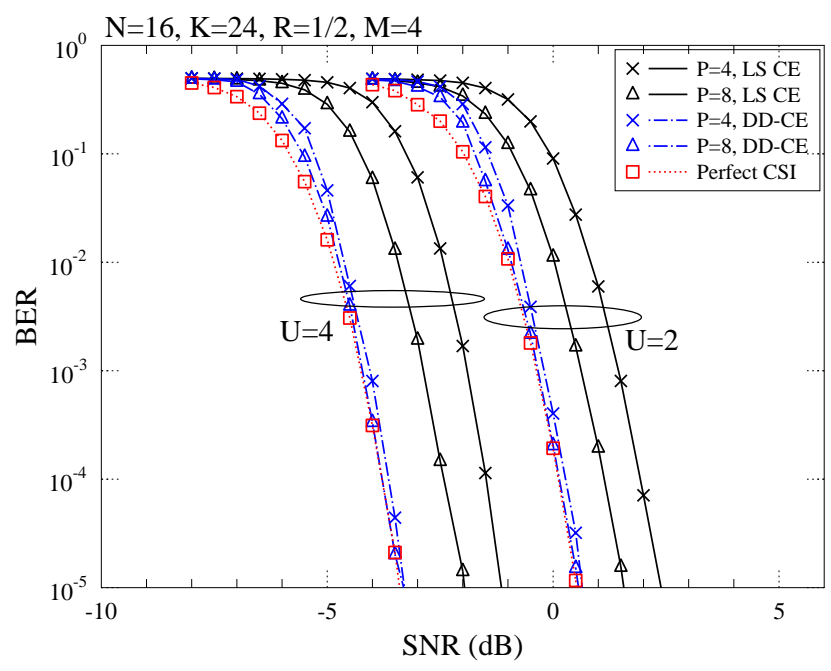

Fig. 12. BER comparison of the joint DD-CE and EC-IDD, and the conventional LS CE combined with our EC-IDD for a $N=16, K=24$ polar-coded SCMA system for communication over quasi-static Rayleigh fading channels under imperfect CSI.

where the normalized user loads are $\lambda=150 \%$ and $200 \%$, respectively, $P=4$ or 8 pilot symbols are employed and the threshold $\phi$ of (33) is chosen to be $\phi=0.8$. Here, the quasi-static Rayleigh fading is assumed for characterizing lowDoppler low-mobility pedestrian scenarios [50,51]. The BER performance of the EC-IDD used in a polar-coded system under perfect CSI is also included as our benchmark. Observe from Figs. 12 and 13 that in the low-SNR region, the CE errors result in a slight performance degradation. By contrast, as the SNR increases, when the DD-CE with 8 pilot symbols is employed, the BER performance of EC-IDD under imperfect CSI approaches that of the perfect-CSI scenario. Furthermore, up to $2 \mathrm{~dB}$ gain can be achieved by our half-rate polar-coded SCMA system having $U=4$ RAs, in the case of $N=16$, 


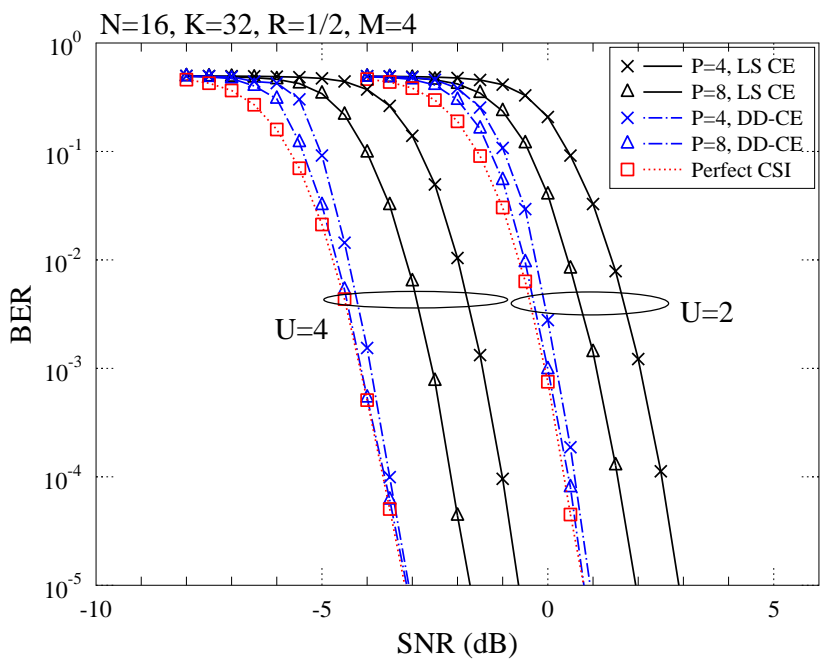

Fig. 13. BER comparison of the proposed joint DD-CE and EC-IDD, and the conventional LS CE combined with our EC-IDD for a $N=16, K=$ 32 polar-coded SCMA system for communication over quasi-static Rayleigh fading channels under imperfect CSI.

$K=32$ when our EC-IDD is embedded into DD-CE over its the EC-IDD counterpart relying on the conventional iterative LS CE using 8 pilot symbols, as shown in Fig. 13.

\section{Complexity}

The complexity of EC-IDD lies mainly in the GA-MP detection and the SLD. According to [31], the number of floating point operations (flops) required to perform the $Z=4$ SLD to decode the $E=256$ half-rate polar code in a single outer iteration is $1.2 \times 10^{4}$. Hence, for decoding a polar-coded SCMA system supporting $K$ users each using $E=256$ half-rate polar code, the decoding complexity $C_{\mathrm{SLD}}$ per outer iteration is expressed as $C_{\mathrm{SLD}}=1.2 \times 10^{4} \mathrm{~K}$. Furthermore, the number of flops required for GA-MP detection within a single outer iteration is calculated as $C_{\mathrm{GA}-\mathrm{MP}}=$ $I F\left\{U N d_{c}\left[15 M+18\left(d_{c}+1\right)\right]+K\left(16 d_{x}+2\right)\right\}$ [52]. Then, the total complexity per iteration per time-slot is expressed in (38).

When EC is employed, the average complexity per iteration of EC-IDD in one time-slot is given in (39), where $K^{(t)}$, $d_{c}^{(t, n)}$ and $d_{x}^{(t, k)}$ are the number of VNs, the number of VNs that share the $n$-th $\mathrm{CN}$ and the number of $\mathrm{CNs}$ that share the $k$-th $\mathrm{VN}$ in the reduced factor graph of Fig. 4(b) in the $t$-th outer iteration, respectively. By contrast, the complexity per iteration of the SCAN decoder and MPA detector that is employed in the state-of-the-art JDD of [34] for a polar-coded SCMA system supporting $K$ users is $C_{\text {SCAN }}=0.8 \times 10^{4} K$ and $C_{\mathrm{MPA}}=F\left\{U N d_{c}\left[\left(7 d_{c}-1\right) M^{d_{c}}+\left(d_{x}-1\right) M\right]\right\}$, respectively, giving a total complexity per iteration expressed in (40).

Fig.14 characterizes the average number of flops required for performing our EC-IDD, the GA-MP-SLD and JDD of [34] per outer iteration in one time-slot supporting different number of users by $N=16$ subcarriers at $\mathrm{SNR}=0 \mathrm{~dB}$. Observe from Fig.14 that while the computational complexity increases

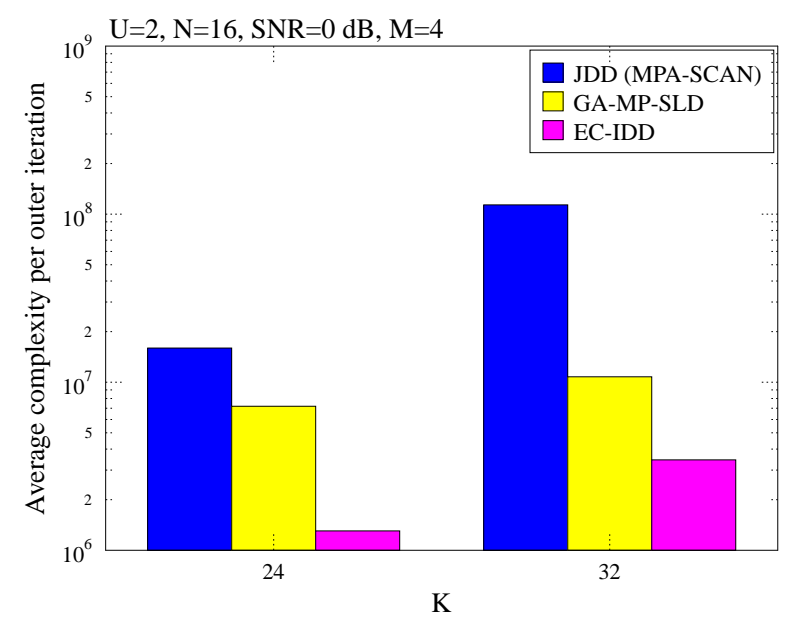

Fig. 14. The average complexity per outer iteration of the JDD [34], GA-MPSLD and the proposed EC-IDD for the polar-coded SCMA system supporting $K=24$ and 32 users within $N=16$ subcarriers at $\mathrm{SNR}=0 \mathrm{~dB}$.

upon increasing $K$, our EC-IDD always imposes significantly lower complexity for both $K=24$ and 32 users than the other two schemes. Explicitly, a $92 \%$ complexity reduction can be observed at a $\lambda=150 \%$ normalized user load at $\mathrm{SNR}=0 \mathrm{~dB}$, compared to the JDD proposed in [34].

\section{CONCLUSION}

A joint iterative receiver design was proposed for the polar-coded SCMA system. First, we have proposed an ECIDD algorithm for jointly and iteratively performing GA-MP detection and SLD at a reduced complexity, while improving the BER performance, compared to the state-of-the-art JDD. Furthermore, we conceived an embedded DD-CE, which does not require an extra loop to exchange information between the detector and channel estimator. Our simulation results demonstrated that the proposed EC-IDD achieves better BER performance than the state-of-the-art JDD in both perfect and imperfect CSI scenarios at a complexity reduction of $92 \%$, when supporting a normalized user load of $150 \%$. Finally, the BER of the proposed embedded DD-CE under imperfect CSI converges to that under perfect CSI.

\section{REFERENCES}

[1] Y. Liu, Z. Qin, M. Elkashlan, Z. Ding, A. Nallanathan, and L. Hanzo, "Non-orthogonal multiple access for 5G and beyond," Proceedings of the IEEE, vol. 105, no. 12, pp. 2347-2381, 2017.

[2] S. Chen, B. Ren, Q. Gao, S. Kang, S. Sun, and K. Niu, "Pattern division multiple access-a novel nonorthogonal multiple access for fifthgeneration radio networks," IEEE Transactions on Vehicular Technology, vol. 66, no. 4, pp. 3185-3196, 2016.

[3] Y. Liu, L.-L. Yang, and L. Hanzo, "Spatial modulation aided sparse code-division multiple access," IEEE Transactions on Wireless Coтmunications, vol. 17, no. 3, pp. 1474-1487, 2018.

[4] L. Dai, B. Wang, Z. Ding, Z. Wang, S. Chen, and L. Hanzo, "A survey of non-orthogonal multiple access for 5G," IEEE Communications Surveys \& Tutorials, vol. 20, no. 3, pp. 2294-2323, 2018.

[5] Y. Liu, L. Yang, P. Xiao, H. Haas, and L. Hanzo, "Spatial modulated multicarrier sparse code-division multiple access," IEEE Transactions on Wireless Communications, vol. 19, pp. 610-623, Jan 2020. 


$$
\begin{aligned}
C_{\mathrm{GA}-\mathrm{MP}-\mathrm{SLD}} & =1.2 \times 10^{4} K+I F\left\{U N d_{c}\left[15 M+18\left(d_{c}+1\right)\right]+K\left(16 d_{x}+2\right)\right\} \\
C_{\mathrm{EC}-\mathrm{IDD}} & =\frac{1}{T} \sum_{t=1}^{T}\left\{1.2 \times 10^{4} K^{(t)}+I F \sum_{n=1}^{N} U d_{c}^{(t, n)}\left[15 M+18\left(d_{c}^{(t, n)}+1\right)\right]+I F \sum_{k=1}^{K}\left(16 d_{x}^{(t, k)}+2\right)\right\} \\
C_{\mathrm{JDD}} & =0.8 \times 10^{4} K+F\left\{U N d_{c}\left[\left(7 d_{c}-1\right) M^{d_{c}}+\left(d_{x}-1\right) M\right]\right\} .
\end{aligned}
$$

[6] Z. Wei, L. Yang, D. W. K. Ng, J. Yuan, and L. Hanzo, "On the performance gain of NOMA over OMA in uplink communication systems," IEEE Transactions on Communications, vol. 68, no. 1, pp. 536-568, 2019.

[7] Y. Liu, L.-L. Yang, and L. Hanzo, "Sparse space-time-frequencydomain spreading for large-scale non-orthogonal multiple access," IEEE Transactions on Vehicular Technology, vol. 69, no. 10, pp. 12327-12332, 2020.

[8] H. Nikopour and H. Baligh, "Sparse code multiple access," in 2013 IEEE 24th Annual International Symposium on Personal, Indoor, and Mobile Radio Communications (PIMRC), pp. 332-336, IEEE, 2013.

[9] C. Zhang, C. Yang, X. Pang, W. Song, W. Xu, S. Zhang, Z. Zhang, and X. You, "Efficient sparse code multiple access decoder based on deterministic message passing algorithm," IEEE Transactions on Vehicular Technology, vol. 69, no. 4, pp. 3562-3574, 2020.

[10] Y.-M. Chen and J.-W. Chen, "On the design of near-optimal sparse code multiple access codebooks," IEEE Transactions on Communications, vol. 68, no. 5, pp. 2950-2962, 2020.

[11] C. Husmann, C. Jayawardena, A. Maaref, P. Xiao, and K. Nikitopoulos, "Low-complexity SCMA detection for unsupervised user access," IEEE Communications Letters, 2020.

[12] C.-P. Wei, H. Yang, C.-P. Li, and Y.-M. Chen, "SCMA decoding via deep learning," IEEE Wireless Communications Letters, 2020.

[13] R. Razavi, A.-I. Mohammed, M. A. Imran, R. Hoshyar, and D. Chen, "On receiver design for uplink low density signature OFDM (LDSOFDM)," IEEE Transactions on Communications, vol. 60, no. 11 , pp. 3499-3508, 2012.

[14] B. Xiao, K. Xiao, S. Zhang, Z. Chen, B. Xia, and H. Liu, "Iterative detection and decoding for SCMA systems with LDPC codes," in 2015 International Conference on Wireless Communications \& Signal Processing (WCSP), pp. 1-5, IEEE, 2015.

[15] F. Wei and W. Chen, "Low complexity iterative receiver design for sparse code multiple access," IEEE Transactions on Communications, vol. 65 no. 2, pp. 621-634, 2016.

[16] B. Tomasi, F. Gabry, V. Bioglio, I. Land, and J.-C. Belfiore, "Low complexity receiver for multi-level polar coded modulation in non orthogonal multiple access," in 2017 IEEE Wireless Communications and Networking Conference Workshops (WCNCW), pp. 1-6, IEEE, 2017.

[17] J. Bao, Z. Ma, M. Xiao, T. A. Tsiftsis, and Z. Zhu, "Bit-interleaved coded SCMA with iterative multiuser detection: Multidimensional constellations design," IEEE Transactions on Communications, vol. 66, no. 11, pp. 5292-5304, 2017.

[18] 3GPP TS 38.212 V15.1.1, "NR Multiplexing and channel coding," 3rd Generation Partnership Project Std. 3GPP, 2018.

[19] Z. B. K. Egilmez, L. Xiang, R. G. Maunder, and L. Hanzo, "The development, operation and performance of the $5 \mathrm{G}$ polar codes," IEEE Communications Surveys \& Tutorials, vol. 22, no. 1, pp. 96-122, 2019

[20] A. Jalali and Z. Ding, "Joint detection and decoding of polar coded 5G control channels," IEEE Transactions on Wireless Communications, vol. 19, no. 3, pp. 2066-2078, 2020.

[21] A. Balatsoukas-Stimming, M. B. Parizi, and A. Burg, "LLR-based successive cancellation list decoding of polar codes," IEEE Transactions on Signal Processing, vol. 63, no. 19, pp. 5165-5179, 2015.

[22] U. U. Fayyaz and J. R. Barry, "Low-complexity soft-output decoding of polar codes," IEEE Journal on Selected Areas in Communications, vol. 32, no. 5, pp. 958-966, 2014.

[23] H. Zhou, X. Tan, W. J. Gross, Z. Zhang, X. You, and C. Zhang, "An improved software list sphere polar decoder with synchronous determination," IEEE Transactions on Vehicular Technology, vol. 68, no. 6, pp. 5236-5245, 2019.

[24] H. Ochiai, P. Mitran, and H. V. Poor, "Capacity-approaching polar codes with long codewords and successive cancellation decoding based on improved Gaussian approximation," IEEE Transactions on Communications, pp. 1-1, 2020.
[25] L. Xiang, Z. B. K. Egilmez, R. G. Maunder, and L. Hanzo, "CRC-aided logarithmic stack decoding of polar codes for ultra reliable low latency communication in 3GPP new radio," IEEE Access, vol. 7, pp. 2855928573, 2019

[26] Z. Babar, Z. B. K. Egilmez, L. Xiang, D. Chandra, R. G. Maunder, S. X. Ng, and L. Hanzo, "Polar codes and their quantum-domain counterparts," IEEE Communications Surveys \& Tutorials, vol. 22, no. 1 pp. 123-155, 2019.

[27] A. Elkelesh, M. Ebada, S. Cammerer, and S. ten Brink, "Decodertailored polar code design using the genetic algorithm," IEEE Transactions on Communications, vol. 67, no. 7, pp. 4521-4534, 2019.

[28] L. Xiang, S. Zhong, R. G. Maunder, and L. Hanzo, "Reduced-complexity low-latency logarithmic successive cancellation stack polar decoding for $5 \mathrm{G}$ new radio and its software implementation," IEEE Transactions on Vehicular Technology, vol. 69, no. 11, pp. 12449-12458, 2020.

[29] C. Pillet, C. Condo, and V. Bioglio, "SCAN list decoding of polar codes," in ICC 2020 - 2020 IEEE International Conference on Communications (ICC), pp. 1-6, 2020.

[30] A. Elkelesh, M. Ebada, S. Cammerer, and S. Ten Brink, "Belief propagation list decoding of polar codes," IEEE Communications Letters, vol. 22, no. 8, pp. 1536-1539, 2018.

[31] L. Xiang, Y. Liu, Z. B. K. Egilmez, R. G. Maunder, L.-L. Yang, and L. Hanzo, "Soft list decoding of polar codes," IEEE Transactions on Vehicular Technology, vol. 69, no. 11, pp. 13921-13926, 2020.

[32] S. Jing, C. Yang, J. Yang, X. You, and C. Zhang, "Joint detection and decoding of polar-coded SCMA systems," in 2017 9th International Conference on Wireless Communications and Signal Processing (WCSP), pp. 1-6, IEEE, 2017.

[33] J. Dai, K. Niu, Z. Si, C. Dong, and J. Lin, "Polar-coded non-orthogonal multiple access," IEEE Transactions on Signal Processing, vol. 66, no. 5 , pp. 1374-1389, 2017.

[34] Z. Pan, E. Li, L. Zhang, J. Lei, and C. Tang, "Design and optimization of joint iterative detection and decoding receiver for uplink polar coded SCMA system," IEEE Access, vol. 6, pp. 52014-52026, 2018.

[35] X. Wu and Z. Wu, "Performance analysis of SCMA system based on polar codes," in 2018 IEEE Globecom Workshops (GC Wkshps), pp. 1-5, IEEE, 2018.

[36] Y. Han, W. Zhou, M. Zhao, and S. Zhou, "Enabling high order SCMA systems in downlink scenarios with a serial coding scheme," IEEE Access, vol. 6, pp. 33796-33809, 2018.

[37] L. Karakchieva and P. Trifonov, "Joint list multistage decoding with sphere detection for polar coded SCMA systems," in SCC 2019; 12th International ITG Conference on Systems, Communications and Coding, pp. 1-6, VDE, 2019.

[38] A. Ghaffari, M. Léonardon, A. Cassagne, C. Leroux, and Y. Savaria, "Toward high-performance implementation of 5G SCMA algorithms," IEEE Access, vol. 7, pp. 10402-10414, 2019.

[39] J. Dai, K. Niu, and J. Lin, "Iterative Gaussian-approximated message passing receiver for MIMO-SCMA system," IEEE Journal of Selected Topics in Signal Processing, vol. 13, no. 3, pp. 753-765, 2019.

[40] P. Zhang, S. Chen, and L. Hanzo, "Embedded iterative semi-blind channel estimation for three-stage-concatenated MIMO-aided QAM turbo transceivers," IEEE Transactions on Vehicular Technology, vol. 63, no. 1 , pp. 439-446, 2013.

[41] J. Jiao, K. Liang, B. Feng, Y. Wang, S. Wu, and Q. Zhang, "Joint channel estimation and decoding for polar coded SCMA system over fading channels," IEEE Transactions on Cognitive Communications and Networking, 2020.

[42] Y. Liu, Z. Tan, H. Hu, L. J. Cimini, and G. Y. Li, "Channel estimation for OFDM," IEEE Communications Surveys \& Tutorials, vol. 16, no. 4 pp. 1891-1908, 2014.

[43] L. Li, Z. Ma, P. Z. Fan, and L. Hanzo, "High-dimensional codebook design for the SCMA down link," IEEE Transactions on Vehicular Technology, vol. 67, no. 10, pp. 10118-10122, 2018. 
[44] T. M. Cover and J. A. Thomas, Elements of Information Theory (Wiley Series in Telecommunications and Signal Processing). USA: WileyInterscience, 2006

[45] F. Wei, W. Chen, Y. Wu, J. Ma, and T. A. Tsiftsis, "Message-passing receiver design for joint channel estimation and data decoding in uplink grant-free SCMA systems," IEEE Transactions on Wireless Communications, vol. 18, no. 1, pp. 167-181, 2018.

[46] W. Yuan, N. Wu, Q. Guo, Y. Li, C. Xing, and J. Kuang, "Iterative receivers for downlink MIMO-SCMA: Message passing and distributed cooperative detection," IEEE Transactions on Wireless Communications, vol. 17, no. 5, pp. 3444-3458, 2018.

[47] Y. Wu, X. Zhu, and A. K. Nandi, "Soft-input turbo channel estimation for single-carrier multiple-input-multiple-output systems," IEEE transactions on vehicular technology, vol. 58, no. 7, pp. 3867-3873, 2009.

[48] M. El-Hajjar and L. Hanzo, "EXIT charts for system design and analysis," IEEE Communications Surveys \& Tutorials, vol. 16, no. 1, pp. 127-153, 2013.

[49] S. X. Ng and L. Hanzo, "On the MIMO channel capacity of multidimensional signal sets," IEEE Transactions on Vehicular Technology, vol. 55, no. 2, pp. 528-536, 2006.

[50] H. Huang, C. B. Papadias, and S. Venkatesan, MIMO communication for cellular networks. Springer Science \& Business Media, 2011.

[51] A. G. i Fabregas and G. Caire, "Coded modulation in the block-fading channel: Coding theorems and code construction," IEEE Transactions on Information Theory, vol. 52, no. 1, pp. 91-114, 2005.

[52] Z. Tang, J. Wang, J. Wang, and J. Song, "A low-complexity detection algorithm for uplink NOMA system based on Gaussian approximation,' in 2017 IEEE Wireless Communications and Networking Conference (WCNC), pp. 1-6, March 2017. 